\title{
External Bifurcations of Double Heterodimensional Cycles with One Orbit Flip
}

\author{
Huimiao Dong, Tiansi Zhang* \\ College of Science, University of Shanghai for Science and Technology, Shanghai, China \\ Email: ${ }^{\star} 17839164984 @ 163 . c o m$
}

How to cite this paper: Dong, H.M. and Zhang, T.S. (2021) External Bifurcations of Double Heterodimensional Cycles with One Orbit Flip. Applied Mathematics, 12, 348-369.

https://doi.org/10.4236/am.2021.124025

Received: March 19, 2021

Accepted: April 27, 2021

Published: April 30, 2021

Copyright (c) 2021 by author(s) and Scientific Research Publishing Inc. This work is licensed under the Creative Commons Attribution International License (CC BY 4.0).

http://creativecommons.org/licenses/by/4.0/ (c) (i) Open Access

\begin{abstract}
In this paper, external bifurcations of heterodimensional cycles connecting three saddle points with one orbit flip, in the shape of " $\infty$ ", are studied in three-dimensional vector field. We construct a poincaré return map between returning points in a transverse section by establishing a locally active coordinate system in the tubular neighborhood of unperturbed double heterodimensional cycles, through which the bifurcation equations are obtained under different conditions. Near the double heterodimensional cycles, the authors prove the preservation of " $\infty$ "-shape double heterodimensional cycles and the existence of the second and third shape heterodimensional cycle and a large 1-heteroclinic cycle connecting with $p_{1}$ and $p_{3}$. The coexistence of a 1-fold large 1-heteroclinic cycle and the " $\infty$ "-shape double heterodimensional cycles and the coexistence conditions are also given in the parameter space.
\end{abstract}

\section{Keywords}

Double Heteroclinic Loops, Orbit Flip, Heteroclinic Bifurcation, Bifurcation Theory

\section{Introduction}

In recent years, bifurcation theory has been widely concerned due to its importance in practical applications (see [1] [2] [3] [4]) and in the study of traveling wave solutions for nonlinear partial differential equations. For example, in 2018, Zilburg and Rosenau [5] studied the qualitative properties of solitons of a dKdV equation,

$$
\partial_{t} u+\partial_{x}\left(u \partial_{x} u\right)+u^{2}=0 .
$$

Then Zhang [6] analyzed (1.1) in the idea of bifurcation theory of dynamical 
system. Roughly to speak, he first set the variable transform $u(x, t)=\phi(x-c t)=\phi(\xi)$ to make system (1.1) be

$$
-c \phi^{\prime}+\left(\phi\left(\phi \phi^{\prime}\right)^{\prime}+\phi^{2}\right)^{\prime}=0,
$$

then integrated (1.2) and got

$$
-c \phi+\phi \phi^{\prime 2}+\phi^{2} \phi^{\prime \prime}+\phi^{2}=g,
$$

where $g$ is the integral constant, and system (1.3) is equivalent to the following regular plane system with $\mathrm{d} \xi=\phi^{2} \mathrm{~d} \tau$

$$
\left\{\begin{array}{l}
\frac{\mathrm{d} \phi}{\mathrm{d} \tau}=y \phi^{2} \\
\frac{\mathrm{d} y}{\mathrm{~d} \tau}=g+c \phi-\phi y^{2}-\phi^{2} .
\end{array}\right.
$$

Clearly the Hamiltonian of system (1.4) is

$$
H(\phi, y)=\frac{1}{2} y^{2} \phi^{2}-g \phi-\frac{1}{2} c \phi^{2}+\frac{1}{3} \phi^{3}=h .
$$

From $\left.H(\phi, y)\right|_{S 1}=h_{1}$, a heteroclinic orbit is found as

$$
y^{2}=\frac{2 h_{1}+2 g \phi+c \phi^{2}-\frac{2}{3} \phi^{3}}{\phi^{2}}
$$

for $g=0, c>0$, and the existing condition is given in some circumstances on two sides of the nonresonant heteroclinic bifurcation.

In fact, different kinds of high co-dimensional homoclinic or heteroclinic bifurcations have been discussed extensively. [7] described a phenomenon that occurred in the bifurcation theory of one-parameter families of diffeomorphisms. If all the equilibrium points of the orbit have the same dimension number of the stable manifold, the heteroclinic cycle is named as an equidimensional loop, otherwise, a heterodimensional. However, since different equilibrium points in $\mathrm{n}$-dimensional systems do not necessarily have stable manifolds of the same dimension, the problem of heterodimensional loop is more general and practical than that of equidimensionals. Jens D.M in [8] considered a self-organized periodic replication process of travelling pulses which has been observed in reaction-Cdiffusion equations, and studied homoclinic orbits near codimension-1 and -2 heteroclinic cycles between an equilibrium and a periodic orbit for ordinary differential equations in three or higher dimensions. Bykov analyzed the bifurcations of systems close to systems having contours composed of separatrices of a pair of saddle points (see [9]). [10] studied the bifurcations of heterodimensional cycles with the connection of two hyperbolic saddle points and strong inclination flip in a four-dimensional system, they presented the conditions for the existence, coexistence and noncoexistence of the heterodimensional orbit, homoclinic orbit and periodic orbit, as well as the co-existence of heterodimensional orbit and homoclinic orbit and obtained some new features from the inclination flip in some bifurcation surfaces. $\mathrm{Xu}$ and $\mathrm{Lu}$ discussed heterodi- 
mensional loop bifurcation with orbit flip and inclination flip respectively in [11] [12] [13], and got the coexistence region of coexisting loop and periodic orbit. Meanwhile, they also constructed an example to provide a good reference for their main bifurcation problems. Specially, Liu's team fabricated a model of heterodimensional cycles to verify their main bifurcation results (see [14] [15] [16] [17]).

However in the study of systems with homoclinic loop or heteroclinic loop, few scholars focused on double heteroclinic bifurcation of three saddle points. We only found that [18] considered the bifurcation problem of rough heteroclinic loops connecting three saddle points, but not a " $\infty$ "-type, for a higher-dimensional system and [19] concerned " $\infty$ "-type double homoclinic loops, but not heteroclinic loops, with resonance characteristic roots in the common case and in a four-dimensional system to obtain the complete bifurcation diagram under different conditions. In this paper, we consider the bifurcation problem of double heteroclinic loops of $\infty$-type connecting three saddle points with four orbits. In addition, we also give an example model to demonstrate the existence of the bifurcation results.

It's worth noting that, in the previous studies about homoclinic and heteroclinic loop bifurcations, few scholars focused on double heterodimensional cycles bifurcations of three saddle points. Jin and Zhu [18] considered the bifurcation problem of rough heteroclinic loop connecting three saddle points in a higher-dimensional system, but the loop is not a " $\infty$ "-type. [20] [21] [22] [23] discussed the heteroclinic loops with two saddle points, but the loops are not heterodimensional cycles. Lu and Liu et al. [10] [11] [13] studied the heterodimensional cycle, but the cycle is also neither a " $\infty$ "-type nor double. Jin et al. [19] [24] considered " $\infty$ "-type double homoclinic loops, but the loops are not heteroclinic or do not connect with three saddle points. Since heterodimensional or heteroclinic cycles are very normal and have applications in solitary wave problems and biology systems, see Kalyan Manna et al. [25] for example, and also for the completeness of theoretical research of heteroclinic bifurcation, in this paper, we focus on the double heterodimensional cycles in $\infty$-type with three saddle points.

The rest of the paper is structured as follows. In Section 2, through establishing a local moving frame system near the unperturbed heterodimensional cycle to obtain the Poincare map and the successor function, we induce the bifurcation equations by using the implicit function theorem. Section 3 will show the bifurcation results on different parameter regions by analyzing the bifurcation equation.

The $C^{r}$ system to be studied is

$$
\dot{Z}=f(z)+g(z, \mu),
$$

where $z \in R^{3}, \mu \in R^{l}, l>4,|\mu| \ll 1, f, g \in C^{r}, r \geq 4$. Specially, when $\mu=0$, the unperturbed system associated with (1.6) is

$$
\dot{Z}=f(z) \text {. }
$$

satisfies the following hypotheses. 
$\left(\mathrm{H}_{1}\right)$ (Hyperbolic) $\mathrm{z}=p_{i}(i=1,2,3)$ are hyperbolic critical points of (1.7) such that $g\left(p_{i}, \mu\right)=g\left(p_{i}, \mathbf{0}\right)=\mathbf{0}$ for all $i$, and $\operatorname{dim}\left(W_{1}^{s}\right)=\operatorname{dim}\left(W_{2}^{u}\right)=\operatorname{dim}\left(W_{3}^{u}\right)=2$ where $\mathbf{0}$ means a zero vector. In addition, the linearization matrix $D_{z} f\left(p_{i}\right)$ has a simple real eigenvalues: $-\rho_{i}^{2},-\rho_{i}^{1}, \lambda_{i}^{1}(i=1,3),-\rho_{2}^{1}, \lambda_{2}^{1}, \lambda_{2}^{2}$ satisfying

$$
-\rho_{i}^{2}<-\rho_{i}^{1}<0<\lambda_{i}^{1},-\rho_{2}^{1}<0<\lambda_{2}^{1}<\lambda_{2}^{2}
$$

Throughout the paper we assume that system (1.7) is of at least $C^{3}$ uniformly linearizable. What's more, there is a small neighborhood $U_{i}(i=1,2,3)$ of the equilibrium $p_{i}$ and a $C^{3}$ diffeomorphism depending on the parameter in $C^{3}$ manner, then we can use successively straightening transformations including the straightening of some orbit segments such that system (1.7) has the following $C^{k}$ normal in $U_{i}$ : as $z=(x, y, v)^{*} \in U_{i}, i=1,3$

$$
\begin{aligned}
& \dot{x}=\left[\lambda_{i}^{1}(\mu)+o(1)\right] x, \\
& \dot{y}=\left[-\rho_{i}^{1}(\mu)+o(1)\right] y+\mathrm{O}(v)(\mathrm{O}(x)+\mathrm{O}(v)), \\
& \dot{v}=\left[-\rho_{i}^{2}(\mu)+o(1)\right] v+\mathrm{O}(y)(\mathrm{O}(x)+\mathrm{O}(y)),
\end{aligned}
$$

and as $z=(x, y, u)^{*} \in U_{2}$

$$
\begin{aligned}
& \dot{x}=\left[\lambda_{2}^{1}(\mu)+o(1)\right] x+\mathrm{O}(u)(\mathrm{O}(y)+\mathrm{O}(u)) \\
& \dot{y}=\left[-\rho_{2}^{1}(\mu)+o(1)\right] y, \\
& \dot{u}=\left[\lambda_{2}^{1}(\mu)+o(1)\right] u+\mathrm{O}(x)(\mathrm{O}(x)+\mathrm{O}(y)),
\end{aligned}
$$

where $k \geq r-2$, the sign “*” stands for transposition. For $\|u\|$ sufficiently small, where $\lambda_{i}^{1}(\mu)=\lambda_{i}^{1}, \quad \rho_{i}^{1}(\mu)=\rho_{i}^{1}, \quad \rho_{i}^{2}(\mu)=\rho_{i}^{2}(i=1,3), \quad \lambda_{2}^{1}(\mu)=\lambda_{2}^{1}$, $\lambda_{2}^{1}(\mu)=\lambda_{2}^{1}, \quad \rho_{2}^{1}(\mu)=\rho_{2}^{1}$ is the corresponding eigenvalues of the linearization matrix of perturbed system (1.6).

$\left(\mathrm{H}_{2}\right)$ (non-degeneration) System (1.7) has a double heterodimensional cycles $\gamma=\gamma_{1}(t) \cup \gamma_{2}(t) \bigcup \gamma_{3}(t) \bigcup \gamma_{4}(t)$, where $\Gamma_{i}=\left\{z=\gamma_{i}(t): t \in R\right\}$, $\gamma_{1}(+\infty)=r_{2}(-\infty)=p_{1}, \quad \gamma_{1}(+\infty)=\gamma_{2}(-\infty)=\gamma_{3}(-\infty)=\gamma_{4}(+\infty)=p_{2}$, $\gamma_{3}(+\infty)=\gamma_{4}(-\infty)=p_{3}$, and

$$
\begin{array}{ll}
\operatorname{dim}\left(T_{\gamma_{1}(t)} W_{1}^{u} \cap T_{\gamma_{1}(t)} W_{2}^{s}\right)=1, & \operatorname{dim}\left(T_{\gamma_{2}(t)} W_{2}^{u} \cap T_{\gamma_{2}(t)} W_{1}^{s}\right)=1, \\
\operatorname{dim}\left(T_{\gamma_{3}(t)} W_{2}^{u} \cap T_{\gamma_{3}(t)} W_{3}^{s}\right)=1, & \operatorname{dim}\left(T_{\gamma_{4}(t)} W_{3}^{u} \cap T_{\gamma_{4}(t)} W_{2}^{s}\right)=1,
\end{array}
$$

Here $\gamma_{i}(t)$ represents the flow of system (17), $t \in R$ and by $T_{q} M$ we denote the tangent space of the manifold $M$ at $q$.

$\left(\mathrm{H}_{3}\right)$ (Orbit flip) Let $e_{i}^{\mp}=\lim _{t \rightarrow \mp \infty} \dot{\gamma}_{i}(-t) /\left|\dot{\gamma}_{i}(-t)\right|$, then

$$
\begin{aligned}
& e_{1}^{+} \in T_{p_{1}} W_{1}^{u}, \quad e_{2}^{+}, e_{3}^{+} \in T_{p_{2}} W_{2}^{u}, \quad e_{4}^{+} \in T_{p_{3}} W_{3}^{u}, \\
& e_{1}^{-}, e_{4}^{-} \in T_{p_{2}} W_{2}^{s}, \quad e_{2}^{-} \in T_{p_{1}} W_{1}^{s}, \quad e_{3}^{-} \in T_{p_{3}} W_{3}^{u},
\end{aligned}
$$

where $e_{i}^{+}, e_{i}^{-}(i=1,2,3)$ are unit eigenvectors corresponding to $\lambda_{i}^{1}$ and $\rho_{i}^{1}(i=1,2,3)$ respectively. Furthermore they satisfy the equation $e_{1}^{-}=-e_{4}^{-}$, $e_{3}^{+}=-e_{2}^{+}$(for details see [19]).

Here, $e_{2}^{+}$and $e_{2}^{-}$are the unit eigenvectors corresponding to $\lambda_{2}^{1}$ and $-\rho_{1}^{2}$ 
which responds $\Gamma_{2}$ enters the equilibrium $p_{1}$ along the strong stable manifold $W_{1}^{s S}$ (as $t \rightarrow+\infty$, enters the equailibruium $p_{2}$ along the unstable manifold $W_{2}^{u}$ (as $t \rightarrow-\infty$ ), that is, from [17], the heteroclinic orbit $\Gamma_{2}$ has orbits flips when $t \rightarrow+\infty$ (see Figure 1 ).

$\left(\mathrm{H}_{4}\right)$ (Strong inclination)

$$
\begin{aligned}
& \lim _{t \rightarrow+\infty} T_{\gamma_{1}(t)} W_{1}^{u}=\operatorname{span}\left\{e_{1}^{-}, e_{2}^{+}\right\}, \quad \lim _{t \rightarrow-\infty} T_{\gamma_{1}(t)} W_{2}^{s}=\operatorname{span}\left\{e_{1}^{+}, e_{2}^{-}\right\} ; \\
& \lim _{t \rightarrow+\infty} T_{\gamma_{2}(t)} W_{2}^{u}=\operatorname{span}\left\{e_{2}^{-}\right\}, \quad \lim _{t \rightarrow-\infty} T_{\gamma_{2}(t)} W_{1}^{s}=\operatorname{span}\left\{e_{2}^{+}\right\} ; \\
& \lim _{t \rightarrow+\infty} T_{\gamma_{3}(t)} W_{2}^{u}=\operatorname{span}\left\{e_{3}^{-}\right\}, \quad \lim _{t \rightarrow-\infty} T_{\gamma_{3}(t)} W_{3}^{s}=\operatorname{span}\left\{e_{3}^{+}\right\} ; \\
& \lim _{t \rightarrow+\infty} T_{\gamma_{4}(t)} W_{3}^{u}=\operatorname{span}\left\{e_{4}^{-}, e_{3}^{+}\right\}, \quad \lim _{t \rightarrow-\infty} T_{\gamma_{4}(t)} W_{2}^{s}=\operatorname{span}\left\{e_{4}^{+}, e_{3}^{-}\right\} .
\end{aligned}
$$

Remark 1.1. Under the assumption $\mathrm{H}_{1}, p_{1}$ and $p_{3}$ have a 1-dimensional unstable manifold and a 2-dimensional stable manifold, while $p_{2}$ has a 2-dimensional unstable manifold and a 1-dimensional stable manifold, hence $\Gamma$ is double heterodimensional cycles.

Remark 1.2. Hypothesis $\left(\mathrm{H}_{4}\right)$ shows that $W_{p_{i}}^{u}$ and $W_{p_{i}}^{s}$ have strong inclination property. Due to the assumption $\left(\mathrm{H}_{2}\right), p_{2}$ has a 2-dimensional unstable manifold, $p_{3}$ has a 2-dimensional stable manifold, and $\operatorname{dim}\left(T_{\gamma_{3}(t)} W_{3}^{u} \cap T_{\gamma_{2}(t)} W_{2}^{s}\right)=1$, we can know the codimension of the heteroclinic orbit $\Gamma_{3}$ is 0 . Then the orbits $\Gamma_{3}$ is transversal, that is, they can be preserved even under small perturbations.

\section{Local Coordinates and Bifurcation Equations}

In this section, we need first to take fundamental solutions of linear variational Equation (see Equation (1.6) as below) and use them as an active coordinate system along the heteroclinic orbits. Then using the new coordinates, we construct the global map spanned by the flow of (1.6) between the sections along the orbits. Next, we set up local maps near equilibriums. Finally the whole Poincaré map can be obtained by composing these maps. The implicit function theorem reveals the bifurcation equation.

By the stable and unstable manifolds theorem and up to two local linear transformations, we see that there are three open neighborhoods $U_{i}$ of $p_{i}=(0,0,0)^{*}$

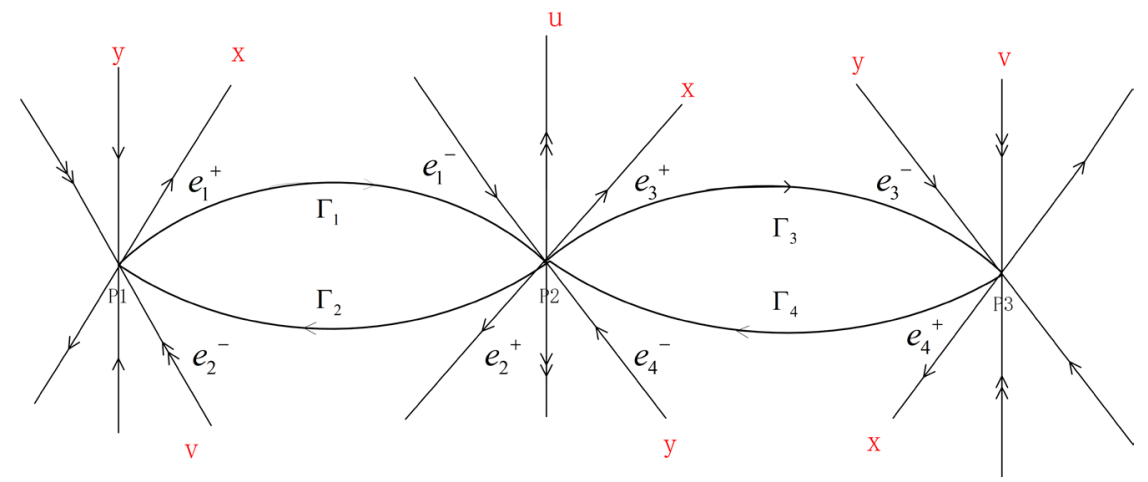

Figure 1. Double heterodimensional cycles of three saddle points $p_{i}$ with four orbits $\gamma_{k}(t)$. 
such that $p_{i}$ have $C^{r-1}$ local manifolds $W_{i, l o c}^{s}$ and $W_{i, l o c}^{s}(i=1,2,3)$ which are expressed as below: for $j=1,3$,

$$
\begin{gathered}
W_{j, l o c}^{u}=\left\{z=(x, y, v)^{*} \in U_{j} \mid(y, v)=(y, v)(x),(y, v)(0)=0, \frac{\partial(y, v)}{\partial x}(0)=0\right\}, \\
W_{j, l o c}^{s}=\left\{z=(x, y, v)^{*} \in U_{j} \mid x=x(y, v), x(0,0)=0, \frac{\partial x}{\partial(y, v)}(0,0)=0\right\}, \\
W_{2, l o c}^{u}=\left\{z=(x, y, u)^{*} \in U_{2} \mid y=y(x, u), y(0,0)=0, \frac{\partial y}{\partial(x, u)}(0,0)=0\right\}, \\
W_{2, l o c}^{s}=\left\{z=(x, y, u)^{*} \in U_{2} \mid(x, u)=(x, u)(y),(x, u)(0)=0, \frac{\partial(x, u)}{\partial y}(0)=0\right\} .
\end{gathered}
$$

Let the coordinate expression of $\gamma_{k}(t)$ be $\gamma_{k}(t)=\left(\gamma_{k}^{x}(t), \gamma_{k}^{y}(t), \gamma_{k}^{v}(t)\right)^{*}$ in the small neighborhood $U_{i}$ of $p_{i},(i=1,3)$, and $\gamma_{k}(t)=\left(\gamma_{k}^{x}(t), \gamma_{k}^{y}(t), \gamma_{k}^{u}(t)\right)^{*}$ in the small neighborhood $U_{2}$ of $p_{2}$. Since $T_{k}>0(k=1,2,3,4)$ is large enough so that $\gamma_{1}\left(-T_{1}\right), \gamma_{2}\left(T_{2}\right) \in U_{1}, \gamma_{3}\left(T_{3}\right), \gamma_{4}\left(-T_{4}\right) \in U_{3}$, $\gamma_{1}\left(T_{1}\right), \gamma_{2}\left(-T_{2}\right), \gamma_{3}\left(-T_{3}\right), \gamma_{4}\left(T_{4}\right) \in U_{2}$ and for $k=1,3,4, \quad \gamma_{k}\left(-T_{k}\right)=(\delta, 0,0)^{*}$, $\gamma_{2}\left(-T_{2}\right)=(-\delta, 0,0)^{*}$, for $k=3,4, \quad \gamma_{k}\left(T_{k}\right)=(0, \delta, 0), r_{1}\left(T_{1}\right)=(0,0, \delta)$, $r_{2}\left(T_{2}\right)=(0,-\delta, 0)$, where $\delta>0$ is small enough.

Now we take into account the linearly variational system and its corresponding adjoint system of (1.7) formed respectively by: let $A_{k}(t)=D f\left(\gamma_{k}(t)\right)$,

$$
\dot{z}=A_{k}(t) z
$$

and

$$
\dot{\phi}=-A_{k}(t)^{*} \phi
$$

Based on the above hypotheses about system (1.7), system (2.1) has exponential dichotomies in $\mathbf{R}^{+}$and $\mathbf{R}^{-}$(see [12]). We can obtain the following properties.

Lemma 2.1. System (2.1) has the fundamental solution matrices

$$
Z_{k}(t)=\left(z_{k}^{1}(t), z_{k}^{2}(t), z_{k}^{3}(t)\right)(k=1,2,3,4)
$$

which satisfy, respectively, for $k=1,4$

$$
\begin{aligned}
& z_{k}^{1}(t), z_{k}^{3}(t) \in\left(T_{\gamma_{k}(t)} \Gamma_{k}(\mu)\right)^{c}, \\
& z_{k}^{2}(t)=\gamma_{k}(t) /\left|\gamma_{k}\left(T_{k}\right)\right| \in T_{\gamma_{k}(t)} W_{k}^{u} \cap T_{\gamma_{k}(t)} W_{k-(-1)^{k}}^{s}
\end{aligned}
$$

that is

$$
Z_{k}\left(-T_{k}\right)=\left(\begin{array}{ccc}
0 & w_{k}^{21} & 0 \\
0 & 0 & 1 \\
1 & 0 & 0
\end{array}\right), \quad Z_{k}\left(T_{k}\right)=\left(\begin{array}{ccc}
w_{k}^{11} & 0 & w_{k}^{31} \\
w_{k}^{12} & (-1)^{k} & w_{k}^{32} \\
w_{k}^{13} & 0 & w_{k}^{33}
\end{array}\right)
$$

where $w_{k}=\left|\begin{array}{ll}w_{k}^{11} & w_{k}^{31} \\ w_{k}^{13} & w_{k}^{33}\end{array}\right| \neq 0,\left|w_{k}^{i 2} \cdot w_{k}^{-1}\right| \ll 1, i=1,3, W_{4}^{u}=W_{2}^{u}$. 
And for $k=2,3$

$$
\begin{aligned}
& z_{k}^{1}(t) \in\left(T_{\gamma_{k}(t)} W_{2}^{u}\right)^{c} \cap T_{\gamma_{k}(t)} W_{k-(-1)^{k}}^{s}, \\
& z_{k}^{2}(t)=\dot{r}_{k}(t) /\left|\dot{\gamma}_{k}\left(-T_{k}\right)\right| \in T_{\gamma_{k}(t)} W_{2}^{u} \cap T_{\gamma_{k}(t)} W_{k-(-1)^{k}}^{s}, \\
& z_{k}^{3}(t) \in T_{\gamma_{k}(t)} W_{2}^{u} \cap\left(T_{\gamma_{k}(t)} W_{k-(-1)^{k}}^{s}\right)^{c},
\end{aligned}
$$

that is,

$$
\begin{aligned}
& Z_{k}\left(-T_{k}\right)=\left(\begin{array}{ccc}
0 & w_{k}^{21} & 0 \\
\bar{w}_{k}^{12} & 0 & 1 \\
1 & 0 & w_{k}^{33}
\end{array}\right) \\
& Z_{2}\left(T_{2}\right)=\left(\begin{array}{ccc}
1 & 0 & w_{2}^{31} \\
w_{2}^{12} & 0 & 1 \\
0 & 1 & 0
\end{array}\right) \\
& Z_{3}\left(T_{3}\right)=\left(\begin{array}{ccc}
1 & 0 & 0 \\
0 & w_{3}^{22} & w_{3}^{32} \\
w_{3}^{13} & 0 & 1
\end{array}\right)
\end{aligned}
$$

where $w_{2}^{21}<0, w_{3}^{22} \neq 0,\left|w_{2}^{12} \cdot w_{2}^{31}\right| \ll 1,\left|w_{3}^{13} \cdot w_{3}^{32}\right| \ll 1,\left|w_{k}^{33} \cdot\left(w_{k}^{21}\right)^{-1}\right| \ll 1, W_{4}^{s}=W_{3}^{u}$.

In what follows, we select $\left(Z_{k}^{2}(t), Z_{k}^{2}(t), Z_{k}^{3}(t)\right)(k=1,2,3,4)$ as a new local coordinate system along $\Gamma_{k}$. Let $\theta_{k}(t)=\left(\phi_{1}(t), \phi_{2}(t), \phi_{3}(t)\right)=\left(Z^{-1}(t)\right)^{*}$ be the fundamental solution matrix of (2.2). By the [1, ?], we can know that the $\phi_{k}^{1}(t)$ is bounded and tends to zero exponentially as $t \rightarrow \pm \infty$.

Take a coordinate transformation

$$
z(t)=h_{k}(t)=\gamma_{k}(t)+Z_{k}(t) N_{k}(t), t \in\left[-T_{k}, T_{k}\right], 0<\varepsilon \ll \delta .
$$

in a small neighborhood of $\Gamma_{k}$, where $N_{k}(t)=\left(n_{k}^{1}(t), 0, n_{k}^{3}(t)\right)^{*}, k=1,2,3,4$, and $n_{k}^{1}(t), n_{k}^{3}(t)$ represents the coordinate decomposition of (1.6) in the new local coordinate system corresponding to $Z_{k}^{1}(t)$ and $Z_{k}^{3}(t)$, Then we can take eight transverse sections vertical to the tangency $T_{\gamma_{k}(t)}$ to each orbit $\gamma_{k}(t)$ (see Figure 2)

$$
\begin{aligned}
& S_{1}^{0}=\left\{z=h_{1}\left(-T_{1}\right):|x|,|y-\delta|,|v-\delta|<\varepsilon\right\}, \\
& S_{2}^{0}=\left\{z=h_{2}\left(-T_{2}\right):-|x|,|y-\delta|,|u-\delta|<\varepsilon\right\},
\end{aligned}
$$

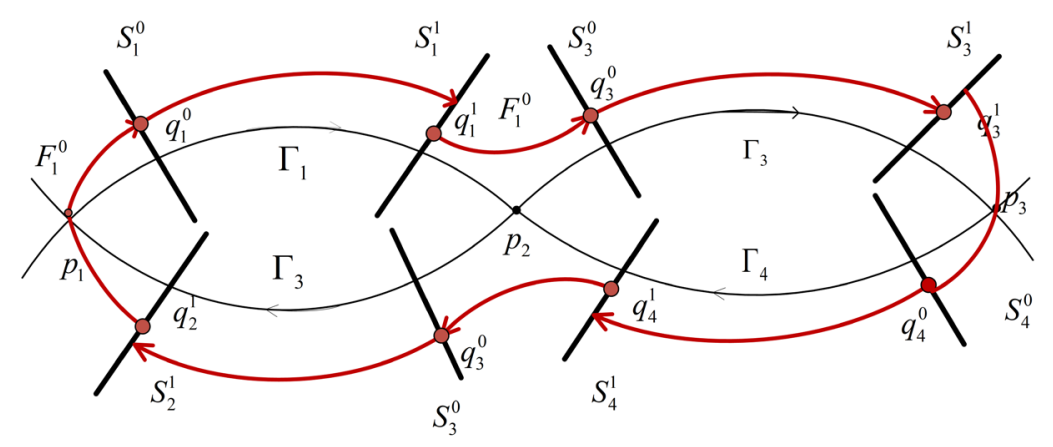

Figure 2. The cross-section and Poincaré map. 


$$
\begin{aligned}
& S_{3}^{0}=\left\{z=h_{3}\left(-T_{1}\right):|x|,|y-\delta|,|u-\delta|<\varepsilon\right\}, \\
& S_{4}^{0}=\left\{z=h_{4}\left(-T_{4}\right):|x|,|y-\delta|,|v-\delta|<\varepsilon\right\}, \\
& S_{1}^{1}=\left\{z=h_{1}\left(T_{1}\right):|x-\delta|,-|y|,|u-\delta|<\varepsilon\right\}, \\
& S_{2}^{1}=\left\{z=h_{2}\left(T_{2}\right):|x-\delta|,|y-\delta|,|v|<\varepsilon\right\}, \\
& S_{3}^{1}=\left\{z=h_{3}\left(T_{3}\right):|x-\delta|,|y|,|v-\delta|<\varepsilon\right\}, \\
& S_{4}^{1}=\left\{z=h_{4}\left(T_{4}\right):|x-\delta|,|y|,|u-\delta|<\varepsilon\right\}
\end{aligned}
$$

In order to obtain the corresponding bifurcation equation, we need to restrict our attention to set up the Poincaré return map of system (1.6). Firstly, we find the relationship between the old coordinates

$$
q_{k}^{0}\left(x_{k}^{0}, y_{k}^{0}, \bar{u}_{k}^{0}\right), \quad q_{j}^{1}\left(x_{j}^{0}, y_{j}^{0}, \bar{u}_{k}^{o}\right)
$$

and new coordinates

$$
q_{i}^{0}\left(n_{i}^{0,1}, 0, n_{i}^{0,3}\right), \quad q_{i}^{1}\left(n_{i}^{1,1}, 0, n_{i}^{1,3}\right)
$$

where $k=2,3, j=1,4, \bar{u}_{k}^{0}=u_{k}^{0} ; k=1,4, j=2,3, \bar{u}_{k}^{0}=v_{k}^{0}$. Then, combining with the Equations (2.3), (2.4), we obtain for $k=1,4$

$$
\left\{\begin{array}{l}
n_{k}^{0,1}=v_{k}^{0} \\
n_{k}^{0,3}=y_{k}^{0} \\
x_{k}^{0}=\delta
\end{array}\right.
$$

and

$$
\left\{\begin{array}{l}
n_{k}^{1,1}=w_{k}^{-1}\left(w_{k}^{33} x_{k}^{1}-w_{k}^{31} u_{k}^{1}\right) \\
n_{k}^{1,3}=w_{k}^{-1}\left(w_{k}^{11} u_{k}^{1}-w_{k}^{13} x_{k}^{1}\right) \\
y_{k}^{1}=\delta+w_{k}^{-1}\left(w_{k}^{12} w_{k}^{33}-w_{k}^{32} w_{k}^{13}\right) x_{k}^{1}+w^{-1}\left(w_{k}^{31} w_{k}^{11}-w_{k}^{12} w_{k}^{31}\right) u_{k}^{1} \approx \delta
\end{array}\right.
$$

for $k=2,3$

$$
\left\{\begin{array}{l}
n_{k}^{0,1}=u_{k}^{0}-w_{k}^{33} y_{k}^{0} \\
n_{k}^{0,3}=y_{k}^{0}-w_{k}^{12} u_{k}^{0} \\
x_{k}^{0}=(-1)^{k-1} \delta
\end{array}\right.
$$

and

$$
\left\{\begin{array}{l}
n_{2}^{1,1}=x_{2}^{1}-w_{2}^{31} y_{2}^{1} \\
n_{2}^{1,3}=y_{2}^{1}-w_{2}^{12} x_{2}^{1} \\
v_{2}^{1}=\delta
\end{array},\left\{\begin{array}{l}
n_{3}^{1,1}=x_{3}^{1}-w_{3}^{32} v_{3}^{1} \\
n_{3}^{1,3}=v_{3}^{1}-w_{3}^{13} x_{3}^{1} \\
y_{3}^{1}=\delta
\end{array}\right.\right.
$$

Then, under transformation (2.5), system (1.6) has the following form by $\dot{\gamma}(t)=f(\gamma(t))$ and $\dot{Z}_{k}(t)=D f(\gamma(t)) Z_{k}(t)$ :

$$
\dot{N}_{k}(t)=\theta_{k}^{*}(t) g_{\mu}\left(\gamma_{k}(t), 0\right) \mu+\text { h.o.t, }
$$

where $g_{\mu}$ is the partial derivation of $g(z, \mu)$ with respect to $\mu$. To integrate (2.10), we get 


$$
\begin{aligned}
N_{k}\left(T_{k}\right) & =N_{k}\left(-T_{k}\right)+\int_{-T_{k}}^{T_{k}} \theta_{k}^{*}(t) g_{\mu}\left(\gamma_{k}(t), 0\right) \mu \mathrm{d} t+\text { h.o.t. } \\
& \triangleq N_{k}\left(-T_{k}\right)+M_{k}^{j} \mu+\text { h.o.t. }
\end{aligned}
$$

where $\quad M_{k}^{j}(\mu)=\int_{-T_{k}}^{T_{k}} \theta_{k}^{j^{*}}(t) g_{\mu}\left(\gamma_{k}(t), 0\right) \mu \mathrm{d} t(j=1,3 ; k=1,2,3,4) \quad$ are $\quad$ called Melnikov vectors respect to $\mu$.

Which are defined as the global maps $F_{k}^{1}: S_{k}^{0} \rightarrow S_{k}^{1}(k=1,2,3,4)$ with the expression by (2.11) given

$$
\begin{aligned}
& \bar{n}_{k}^{1,1}=n_{k}^{0,1}+M_{k}^{1} \mu+\text { h.o.t., } \\
& \bar{n}_{k}^{1,3}=n_{k}^{0,3}+M_{k}^{3} \mu+\text { h.o.t.. }
\end{aligned}
$$

as follows

$$
F_{k}^{1}\left(n_{k}^{0,1}, 0, n_{k}^{0,3}\right)=\left(\bar{n}_{k}^{1,1}, 0, \bar{n}_{k}^{1,3}\right) .
$$

Next we consider the local maps,

$$
\begin{array}{ll}
F_{1}^{0}: q_{2}^{1} \in S_{2}^{1} \mapsto q_{1}^{0} \in S_{1}^{0}, & F_{2}^{0}: q_{1}^{1} \in S_{1}^{1} \mapsto q_{3}^{0} \in S_{3}^{0}, \\
F_{3}^{0}: q_{3}^{1} \in S_{3}^{1} \mapsto q_{4}^{0} \in S_{4}^{0}, & F_{4}^{0}: q_{4}^{1} \in S_{4}^{1} \mapsto q_{2}^{0} \in S_{2}^{0}
\end{array}
$$

induced by flows confined in the neighborhood $U_{i}(i=1,2,3)$.

Let $\tau_{1}, \tau_{3}$ be the time spent from $q_{2}^{1}$ to $q_{1}^{0}$ and from $q_{3}^{1}$ to $q_{4}^{0}$ respectively, corresponding their Shilnikov time $s_{1}=\mathrm{e}^{-\rho_{1}^{1}(\mu) \tau_{1}}, s_{3}=\mathrm{e}^{-\rho_{3}^{1}(\mu) \tau_{3}}$. Let $\tau_{2}, \tau_{4}$ be the time spent from $q_{1}^{1}$ to $q_{3}^{0}$ and from $q_{4}^{1}$ to $q_{2}^{0}$, then their Shilnikov time are $s_{k}=\mathrm{e}^{\lambda_{2}^{1}(\mu) \tau_{k}}(k=2,4)$.

Then under the assumptions among the eigenvalues, by the normal forms (1.8)-(1.9), and the formula of variation of constants, we obtain the local maps:

$$
\begin{aligned}
& F_{1}^{0}: q_{2}^{1}\left(x_{2}^{1}, y_{2}^{1}, v_{2}^{1}\right) \in S_{2}^{1} \mapsto q_{1}^{0}\left(x_{1}^{0}, y_{1}^{0}, v_{1}^{0}\right) \in S_{1}^{0} \\
& x_{2}^{1}=x\left(T_{2}\right) \approx x_{1}^{0} s_{1}^{\frac{\lambda_{1}^{1}(\mu)}{\rho_{1}^{1}(\mu)}}, \quad y_{1}^{0}=y\left(T_{2}+\tau_{1}\right) \approx y_{2}^{1} s_{1}, \quad v_{1}^{0}=v\left(T_{2}+\tau_{2}\right) \approx v_{2}^{1} s_{1}^{\frac{p_{1}^{2}(\mu)}{\rho_{1}^{1}(\mu)}} . \\
& F_{2}^{0}: q_{1}^{1}\left(x_{1}^{1}, y_{1}^{1}, u_{1}^{1}\right) \in S_{1}^{1} \mapsto q_{3}^{0}\left(x_{3}^{0}, y_{3}^{0}, u_{3}^{0}\right) \in S_{3}^{0} \\
& x_{1}^{1}=x\left(T_{1}\right) \approx x_{3}^{0} s_{2}, \quad y_{3}^{0}=y\left(T_{1}+\tau_{2}\right) \approx y_{1}^{1} s_{2}^{\frac{\rho_{2}^{1}}{\lambda_{2}^{1}}}, \quad u_{1}^{1}=u\left(T_{2}\right) \approx u_{3}^{0} s_{2}^{\lambda_{2}^{2}(\mu)} \\
& F_{3}^{0}: q_{3}^{1}\left(x_{3}^{1}, y_{3}^{1}, v_{3}^{1}\right) \in S_{3}^{1} \mapsto q_{4}^{0}\left(x_{4}^{0}, y_{4}^{0}, v_{4}^{0}\right) \in S_{4}^{0} \\
& x_{3}^{1}=x\left(T_{3}\right) \approx x_{4}^{0} s_{3}^{\frac{\lambda_{3}^{1}(\mu)}{\rho_{3}^{1}(\mu)}}, \quad y_{4}^{0}=y\left(T_{3}+\tau_{3}\right) \approx y_{3}^{1} s_{3}, \quad v_{4}^{0}=v\left(T_{3}+\tau_{3}\right) \approx v_{3}^{1} s_{3}^{\frac{\rho_{3}^{2}(\mu)}{\rho_{3}^{1}(\mu)}} \\
& F_{4}^{0}: q_{4}^{1}\left(x_{4}^{1}, y_{4}^{1}, u_{4}^{1}\right) \in S_{4}^{1} \mapsto q_{2}^{0}\left(x_{2}^{0}, y_{2}^{0}, u_{2}^{0}\right) \in S_{2}^{0} \\
& x_{4}^{1}=x\left(T_{4}\right) \approx x_{2}^{0} s_{4}, \quad y_{2}^{0}=y\left(T_{4}+\tau_{4}\right) \approx y_{4}^{1} s_{4}^{\frac{\rho_{2}^{1}(\mu)}{\lambda_{2}^{1}(\mu)}}, \quad u_{4}^{1}=u\left(T_{4}\right) \approx u_{2}^{0} s_{4}^{\frac{\lambda_{2}^{2}(\mu)}{\lambda_{2}^{1}(\mu)}}
\end{aligned}
$$

Thus, by (2.6), (2.12) (2.13), we obtain the first Poincaré map $F_{1}=F_{1}^{1} \circ F_{1}^{0}: S_{2}^{1} \mapsto S_{1}^{1}$ as follows

$$
\left\{\begin{array}{l}
\bar{n}_{1}^{1,1}=M_{1}^{1} \mu+\text { h.o.t. } \\
\bar{n}_{1}^{1,3}=M_{1}^{3} \mu+\text { h.o.t.; }
\end{array}\right.
$$

by (2.8), (2.12), (2.14), we obtain the Poincaré map $F_{3}=F_{3}^{1} \circ F_{2}^{0}: S_{1}^{1} \mapsto S_{3}^{1}$ as 
follows

$$
\left\{\begin{array}{l}
\bar{n}_{3}^{1,1}=u_{3}^{0}+w_{3}^{33} \delta s_{2}^{\beta_{2}}+M_{3}^{1} \mu+\text { h.o.t. } \\
\bar{n}_{3}^{1,3}=w_{3}^{12} u_{3}^{0}+\delta s_{2}^{\beta_{2}}+M_{3}^{3} \mu+\text { h.o.t. }
\end{array}\right.
$$

by (2.8), (2.12), (2.15), we obtain the Poincaré map $F_{4}=F_{4}^{1} \circ F_{3}^{0}: S_{3}^{1} \mapsto S_{4}^{1}$ as follows

$$
\left\{\begin{array}{l}
\bar{n}_{4}^{1,1}=M_{4}^{1} \mu+\text { h.o.t. } \\
\bar{n}_{4}^{1,3}=M_{4}^{3} \mu+\text { h.o.t. }
\end{array}\right.
$$

by (2.6), (2.12), (2.16), we obtain the Poincaré map $F_{2}=F_{2}^{1} \circ F_{4}^{0}: S_{4}^{1} \mapsto S_{2}^{1}$ as follows

$$
\left\{\begin{array}{l}
\bar{n}_{2}^{1,1}=u_{2}^{0}-w_{2}^{33} \delta s_{4}^{\beta_{2}}+M_{2}^{1} \mu+\text { h.o.t. } \\
\bar{n}_{2}^{1,3}=\delta s_{4}^{\beta_{2}}-w_{2}^{12} u_{2}^{0}+M_{2}^{3} \mu+\text { h.o.t. }
\end{array}\right.
$$

Then, by (2.7), (2.9), (2.17), (2.18), (2.19), (2.20), we induce the successor functions

$$
\begin{aligned}
G & =\left(G_{1}, G_{2}, G_{3}\right)=G\left(G_{1}^{1}, G_{1}^{3}, G_{3}^{1}, G_{3}^{3}, G_{4}^{1}, G_{4}^{3}, G_{2}^{1}, G_{2}^{3}\right) \\
& =G\left(s_{1}, s_{2}, s_{3}, s_{4}, u_{1}^{0}, u_{4}^{0}, v_{1}^{1}, v_{4}^{1}\right) \\
& =\left(F_{1}\left(q_{2}^{1}\right)-q_{1}^{1}, F_{3}\left(q_{1}^{1}\right)-q_{3}^{1}, F_{2}\left(q_{3}^{1}\right)-q_{4}^{1}, F_{2}\left(q_{4}^{1}\right)-q_{2}^{1}\right)
\end{aligned}
$$

where

$$
\begin{gathered}
G_{1}^{1}=w_{1}^{-1} w_{1}^{33} \delta s_{2}^{\frac{\lambda_{2}^{2}}{\lambda_{2}^{1}}}+w_{1}^{-1} w_{1}^{31} u_{3}^{0} s_{2}+M_{1}^{1} \mu+\text { h.o.t.; } \\
G_{1}^{3}=w_{1}^{-1} w_{1}^{11} u_{3}^{0} s_{2}+w_{1}^{-1} w_{1}^{13} \delta s_{2}^{\frac{\lambda_{2}^{2}}{\lambda_{2}^{1}}}+M_{1}^{3} \mu+\text { h.o.t. } \\
G_{2}^{1}=u_{2}^{0}-w_{2}^{33} \delta s_{4}^{\beta_{2}}+w_{2}^{31} y_{2}^{1}+M_{2}^{1} \mu+\text { h.o.t.; } \\
G_{2}^{3}=\delta s_{4}^{\beta_{2}}-w_{2}^{12} u_{2}^{0}-y_{2}^{1}+M_{2}^{3} \mu+\text { h.o.t. } \\
G_{3}^{1}=u_{3}^{0}+w_{3}^{33} \delta s_{2}^{\beta_{2}}+w_{3}^{32} v_{3}^{1}+M_{3}^{1} \mu+\text { h.o.t.; } \\
G_{3}^{3}=w_{3}^{12} u_{3}^{0}+\delta s_{2}^{\beta_{2}}-v_{3}^{1}+M_{3}^{3} \mu+\text { h.o.t. } \\
G_{4}^{1}=w_{4}^{-1} w_{4}^{33} \delta s_{4}^{\frac{\lambda_{2}^{2}}{\lambda_{2}^{1}}}+w_{4}^{-1} w_{4}^{31} u_{2}^{0} s_{4}+M_{4}^{1} \mu+\text { h.o.t.; } \\
G_{4}^{3}=-w_{4}^{-1} w_{4}^{11} u_{2}^{0} s_{4}+w_{4}^{-1} w_{4}^{13} \delta s_{4}^{\frac{\lambda_{2}^{2}}{\lambda^{1}}}+M_{4}^{3} \mu+\text { h.o.t. }
\end{gathered}
$$

By the implicit function theorem, solving the equation $\left(G_{2}^{1}, G_{2}^{3}, G_{3}^{1}, G_{3}^{3}\right)=0$, we have

$$
\begin{aligned}
& u_{2}^{0}=\left(w_{2}^{31}-w_{2}^{33}\right) \delta s_{4}^{\beta_{2}}-w_{2}^{31} M_{2}^{3} \mu-M_{2}^{1} \mu+\text { h.o.t. } \\
& y_{2}^{1}=\delta s_{4}^{\beta_{2}}-w_{2}^{12} M_{2}^{1} \mu-M_{2}^{3} \mu+\text { h.o.t. } \\
& u_{3}^{0}=-\left(w_{3}^{32}+w_{3}^{33}\right) \delta s_{2}^{\beta_{2}}-w_{3}^{32} M_{3}^{3} \mu-M_{3}^{1} \mu+\text { h.o.t. } \\
& v_{3}^{1}=\delta s_{2}^{\beta_{2}}-w_{3}^{12} M_{3}^{1} \mu+M_{3}^{3} \mu+\text { h.o.t. }
\end{aligned}
$$

Substituting them into $\left(G_{4}^{1}, G_{4}^{3}, G_{1}^{1}, G_{1}^{3}\right)=0$, we obtain the bifurcation equations, for $w_{1}^{13} \neq 0, w_{1}^{33} \neq 0, w_{4}^{13} \neq 0, w_{4}^{33} \neq 0$ 


$$
\left\{\begin{array}{l}
w_{4}^{33} \delta s_{4}^{\frac{\lambda_{2}^{2}}{\lambda_{2}^{1}}}-w_{4}^{31} s_{4}\left(w_{2}^{31} M_{2}^{3} \mu+M_{2}^{1} \mu\right)+w_{4} M_{4}^{1} \mu+\text { h.o.t. } \\
w_{4}^{13} \delta s_{4}^{\frac{\lambda_{2}^{2}}{\lambda_{2}^{1}}}+w_{4}^{11} s_{4}\left(w_{2}^{31} M_{2}^{3} \mu+M_{2}^{1} \mu\right)+w_{4} M_{4}^{3} \mu+\text { h.o.t. } \\
w_{1}^{33} \delta s_{2}^{\frac{\lambda_{2}^{2}}{\lambda_{2}^{1}}}-w_{1}^{31} s_{2}\left(w_{3}^{32} M_{3}^{3} \mu+M_{3}^{1} \mu\right)+w_{1} M_{1}^{1} \mu+\text { h.o.t. } \\
w_{1}^{13} \delta s_{2}^{\frac{\lambda_{2}^{2}}{\lambda_{2}^{1}}}-w_{1}^{11} s_{2}\left(w_{3}^{32} M_{3}^{3} \mu+M_{3}^{1} \mu\right)+w_{1} M_{1}^{3} \mu+\text { h.o.t. }
\end{array}\right.
$$

Remark 2.1. In fact, $M_{i}^{j}$ is independent of the choice of $T_{i}$ for $i=1,2,3,4, j=1,3$, which can be verified similarly as in [13]

Remark 2.2. Generally, in two-dimensional plane system, when we study bifurcations of singular cycle, Poincaré mapping can only be established on one side of the singular cycle. Therefore, there are no other types of orbits except the one with infinite approaching to saddle point on the left side of $p_{1}$ and the right side of $p_{3}$. However, in high-dimensional system, it remains to be verified whether other types of orbits can bypass different surfaces for connection. To make the study go on, we assume that $s_{1}=s_{3}=0$, that is, the orbit starting from $S_{2}^{1}$ to $S_{1}^{0}$ just be a singular orbit which is infinitely approaching $p_{1}$ when $t \rightarrow \pm \infty$; for the orbit starting from $S_{3}^{1}$ to $S_{4}^{0}$ is similar near $p_{3}$.

Remark 2.3. Basing on remark 2.2, it can be seen that (2.13) and (2.14) become $F_{1}^{0}: q_{2}^{1}\left(0, y_{2}^{1}, \delta\right) \in S_{2}^{1} \mapsto q_{1}^{0}(\delta, 0,0) \in S_{1}^{0}$ and $F_{3}^{0}: q_{3}^{1}\left(0, \delta, v_{3}^{1}\right) \in S_{3}^{1} \mapsto q_{4}^{0}(\delta, 0,0) \in S_{4}^{0}$.

Remark 2.4. Shilnikov variables were introduced by Shilnikov in 1968 to compute the local transition map near equilibria to leading order. Instead of solving an initial-value problem, solutions near the equilibrium are found using an appropriate boundary-value problem.

\section{Heterodimensional Cycle Bifurcation of " $\infty$ " Type}

In this section, we analyze the bifurcation of system (1.6) under hypotheses $\left(A_{1}\right)-\left(A_{4}\right)$. The existence of " $\infty$ "-shape double heterodimensional cycles, the heteroclinic cycle composed of three orbits and connecting with three saddle points, and large 1-heteroclinic connecting with $p_{1}$ and $p_{3}$ are studied by discussing the corresponding bifurcation equation. Clearly if $s_{2}=s_{4}=0$ the double heterodimensional cycle (" $\infty$ ") of system (1.6) is persistent; if $s_{4}=0$, $s_{2}>0$, system (1.6) has a heterodimensional cycle consisting of two saddles of (1.2) type and one saddle of (2.1) type composed of one big orbit linking $p_{3}, p_{1}$ and two orbits linking $p_{3}, p_{2}$ and $p_{2}, p_{1}$ respectively, which is called the second shape heterodimensional cycle in later of this paper; if $s_{2}=0, s_{4}>0$, system (1.6) has another heterodimensional cycle consisting of two saddles of (2.1) type and one saddle of (1.2) type composed of one big orbit linking $p_{1}, p_{3}$ and two orbits linking $p_{1}, p_{2}$ and $p_{2}, p_{3}$ respectively, which is called another second shape heterodimensional cycle in later of this paper; if $s_{2}>0$ and $s_{4}>0$, system (1.6) has the large 1-heteroclinic cycle consisting of two saddles 
$p_{1}$ and $p_{3}$ of (2.1) type composed with two big orbits linking $p_{1}, p_{3}$ and $p_{3}, p_{1}$ respectively. What is noteworthy is that if the conditions make $s_{2}>0$ untenable and set $s_{4}=0$ tenable, the conditions make $s_{4}>0$ untenable and $S_{2}=0$ tenable, system (1.6) has the third heterodimensional cycle consisting of one saddle $p_{2}$ of (2.1) type and one saddle $p_{3}$ of (1.2) type and composed of one orbit starting from $p_{2}$ to $p_{3}$ and another orbit starting from $p_{3}$ to $p_{2}$ under the assumption $\left(\mathrm{H}_{2}\right)$. So in the following, we need to consider solutions $s_{2}$ and $s_{4}$ of the bifurcation Equation (2.21).

\subsection{Analysis Procedure}

Corresponding results about the existence of the second heterodimensional cycle, the third heterodimensional cycle and large-1 heteroclinic cycle, as well as the coexistence of double heterodimensional cycle and the large 1-heteroclinic cycle are contained in the next theorems. For convenience to discuss, we set eight regions:

$$
\begin{aligned}
& N_{+}^{+}=\left\{\mu \mid w_{1}^{31}\left(w_{3}^{32} M_{3}^{3} \mu+M_{3}^{1} \mu\right)>0, w_{1} M_{1}^{1} \mu<0\right\}, \\
& N_{+}^{-}=\left\{\mu \mid w_{1}^{31}\left(w_{3}^{32} M_{3}^{3} \mu+M_{3}^{1} \mu\right)>0, w_{1} M_{1}^{1} \mu>0\right\} \\
& N_{-}^{+}=\left\{\mu \mid w_{1}^{31}\left(w_{3}^{32} M_{3}^{3} \mu+M_{3}^{1} \mu\right)<0, w_{1} M_{1}^{1} \mu<0\right\}, \\
& N_{-}^{-}=\left\{\mu \mid w_{1}^{31}\left(w_{3}^{32} M_{3}^{3} \mu+M_{3}^{1} \mu\right)<0, w_{1} M_{1}^{1} \mu>0\right\} ; \\
& B_{+}^{+}=\left\{\mu \mid w_{4}^{31}\left(w_{2}^{32} M_{2}^{3} \mu+M_{2}^{1} \mu\right)>0, w_{4} M_{4}^{1} \mu<0\right\}, \\
& B_{+}^{-}=\left\{\mu \mid w_{4}^{31}\left(w_{2}^{32} M_{2}^{3} \mu+M_{2}^{1} \mu\right)>0, w_{4} M_{4}^{1} \mu>0\right\}, \\
& B_{-}^{+}=\left\{\mu \mid w_{4}^{31}\left(w_{2}^{32} M_{2}^{3} \mu+M_{2}^{1} \mu\right)<0, w_{4} M_{4}^{1} \mu<0\right\}, \\
& B_{-}^{-}=\left\{\mu \mid w_{4}^{31}\left(w_{2}^{32} M_{2}^{3} \mu+M_{2}^{1} \mu\right)<0, w_{4} M_{4}^{1} \mu>0\right\}
\end{aligned}
$$

From the discussion of Theorem 1 , if one of $s_{2}$ and $s_{4}$ is 0 , the second heterodimensional cycle will appear. And if $s_{2}>0, s_{4}>0$, a large 1-heteroclinic cycle connecting with $p_{1}$ and $p_{3}$ will exist. As well as, if there are conditions that make $s_{2}>0$ be invalid and $s_{4}=0$ or $s_{4}>0$ be invalid and $s_{2}=0$, the third heterodimensional cycle will arise. Therefore it is enough to discuss the solutions $s_{i}(i=2,4)$ of the Equation (2.21).

Since the first two equations of Equation (2.11) have the same structure as the last two, we only analyze the first and second equations as following

$$
\left\{\begin{array}{l}
w_{4}^{33} \delta s_{4}^{\frac{\lambda_{2}^{2}}{\lambda_{2}^{1}}}-w_{4}^{31} s_{4}\left(w_{2}^{31} M_{2}^{3} \mu+M_{2}^{1} \mu\right)+w_{4} M_{4}^{1} \mu+\text { h.o.t. }=0 \\
w_{4}^{13} \delta s_{4}^{\frac{\lambda_{2}^{2}}{\lambda_{2}^{1}}}+w_{4}^{11} s_{4}\left(w_{2}^{31} M_{2}^{3} \mu+M_{2}^{1} \mu\right)+w_{4} M_{4}^{3} \mu+\text { h.o.t. }=0
\end{array}\right.
$$

Set $\alpha=\frac{\lambda_{2}^{2}}{\lambda_{2}^{1}}$, rewrite the first Equation of (3.1) as

$$
w_{4}^{31}\left(w_{2}^{31} M_{2}^{3} \mu+M_{2}^{1} \mu\right) s_{4}-w_{4} M_{4}^{1} \mu-w_{4}^{33} \delta s_{4}^{\alpha}+\text { h.o.t. } \triangleq L\left(s_{4}, \mu\right)-N\left(s_{4}, \mu\right)=0,
$$


where

$$
L\left(s_{4}, \mu\right)=w_{4}^{31}\left(w_{2}^{31} M_{2}^{3} \mu+M_{2}^{1} \mu\right) s_{4}-w_{4} M_{4}^{1} \mu, \quad N\left(s_{4}, \mu\right)=w_{4}^{33} \delta s_{4}^{\alpha}+\text { h.o.t. }
$$

Then we have

$$
\begin{gathered}
L(0, \mu)-N(0, \mu)=-w_{4} M_{4}^{1} \mu+\text { h.o.t. } \\
L_{s_{4}}^{\prime}\left(s_{4}, \mu\right)-N_{s_{4}}^{\prime}\left(s_{4}, \mu\right)=w_{4}^{31}\left(w_{2}^{31} M_{2}^{3} \mu+M_{2}^{1} \mu\right)-w_{4}^{33} \alpha \delta s_{4}^{\alpha-1}+\text { h.o.t. }
\end{gathered}
$$

If $\left(w_{4}^{33}\right)^{-1} w_{4}^{31}\left(w_{2}^{31} M_{2}^{3} \mu+M_{2}^{1} \mu\right)>0$, the equation $L_{s_{4}}^{\prime}\left(s_{4}, \mu\right)-N_{s_{4}}^{\prime}\left(s_{4}, \mu\right)=0$ has a unique small positive solution $\tilde{s}_{4}=\left(w_{4}^{31}\left(w_{4}^{33} \alpha \delta\right)^{-1}\left(w_{2}^{31} M_{2}^{3} \mu+M_{2}^{1} \mu\right)\right)^{\frac{1}{\alpha-1}}+$ h.o.t. If $\left(w_{4}^{33}\right)^{-1} w_{4}^{31}\left(w_{2}^{31} M_{2}^{3} \mu+M_{2}^{1} \mu\right)<0$, it makes $\tilde{s}_{4}>0$ be untenable.

1) If $w_{4}^{33}>0, \mu \in B_{-}^{-}$or $w_{4}^{33}<0, \mu \in B_{+}^{+}$, the straight line $L$ and the curve $N$ cannot intersect in the half plane for $s_{4}>0$, so Equation (3.2) has not any positive solutions, that is, system (1.6) only has the transversal heteroclinic orbit $\gamma_{3}(t)$ in the region $\Gamma_{2} \cup \Gamma_{3}$.

2) If $w_{4}^{33}>0, \mu \in B_{-}^{+}$or $w_{4}^{33}<0, \mu \in B_{+}^{-}$, the straight line $L\left(t_{4}, \mu\right)$ and the curve $N\left(s_{4}, \mu\right)$ intersect at one positive point, that is, (3.2) has one positive solution.

Without loss of generality, we discuss the case $w_{4}^{33}>0, \mu \in B_{-}^{+}$. There are

$$
L(0, \mu)>N(0, \mu), L_{s_{4}}^{\prime}\left(s_{4}, \mu\right)<N_{s_{4}}^{\prime}\left(s_{4}, \mu\right), L\left(\bar{s}_{4}, \mu\right)-N\left(\bar{s}_{4}, \mu\right)=-w_{4}^{33} \delta \bar{s}_{4}^{\alpha}<0,
$$

where $\bar{s}_{4}=\frac{w_{4} M_{4}^{1} \mu}{w_{4}^{31}\left(w_{2}^{31} M_{2}^{3} \mu+M_{2}^{1} \mu\right)}$.

When $\left|M_{4}^{1} \mu\right|=o\left(\left|w_{2}^{31} M_{2}^{3} \mu+M_{2}^{1} \mu\right|\right), 0<\bar{s}_{4} \ll 1$. It is clear that (3.2) has a unique solution $s_{4}^{1^{*}}$ satisfying $0<s_{4}^{1^{*}}<\bar{S}_{4} \ll 1$. Putting it into the second equation of (3.1), there is $w_{4}^{13} \delta\left(s_{4}^{1^{*}}\right)^{\frac{\lambda_{2}^{2}}{\lambda_{2}^{1}}}+w_{4}^{11}\left(w_{2}^{31} M_{2}^{3} \mu+M_{2}^{1} \mu\right) s_{4}^{1^{*}}+w_{4} M_{4}^{3} \mu+$ h.o.t. $=0$, it defines a surface

$$
L_{4}^{1}(\mu)=\left\{\mu: w_{4}^{13} \delta\left(\frac{w_{4} M_{4}^{1} \mu}{w_{2}^{31} w_{4}^{31} M_{2}^{3} \mu+w_{4}^{31} M_{2}^{1} \mu}\right)^{\frac{\lambda_{2}^{2}}{\lambda_{2}^{1}}}+w_{4}^{11}\left(w_{4}^{31}\right)^{-1} w_{4} M_{4}^{1} \mu+w_{4} M_{4}^{3} \mu+\text { h.o.t. }=0\right\},
$$

with a normal surface $\Sigma=\operatorname{span}\left\{M_{4}^{3}\right\}$ at $\mu=0$ for $M_{4}^{3} \mu>0$. That is to say, system (1.6) has the only one heteroclinic orbit $\gamma_{1}(t)$ consisting of $p_{1}$ and $p_{3}$ near $\Gamma_{1} \cup \Gamma_{3}$ for $\mu \in L_{4}^{1}(\mu)$.

3) If $w_{4}^{33}>0, \mu \in B_{+}^{+}$or $w_{4}^{33}<0, \mu \in B_{-}^{-}$, there are two special cases:

a) As $\left|\left(w_{2}^{31} M_{2}^{3} \mu+M_{2}^{1} \mu\right)\right| s_{4} \ll \max \left\{s_{4}^{\alpha},\left|M_{4}^{1} \mu\right|\right\}$, Equation (3.2) can be simplified to be

$$
w_{4} M_{4}^{1} \mu+w_{4}^{33} \delta \bar{s}_{4}^{\alpha}+\text { h.o.t. }=0 .
$$

It has a solution $s_{4}^{2^{*}}=\left(-\frac{w_{4} M_{4}^{1} \mu}{w_{4}^{33} \delta}\right)^{\frac{1}{\alpha}}+$ h.o.t. Substituting $s_{4}^{2^{*}}$ into the second equation of (2.11), we get immediately a surface $L_{4}^{2}(\mu)$ tangent to $L_{14}(\mu)$, 


$$
L_{4}^{2}(\mu)=\left\{\mu: w_{4}^{11}\left(-\frac{w_{4} M_{4}^{1} \mu}{w_{4}^{33} \delta}\right)^{\frac{\lambda_{2}^{1}}{\lambda_{2}^{2}}}\left(w_{2}^{31} M_{2}^{3} \mu+M_{2}^{1} \mu\right)-w_{4}^{13}\left(w_{4}^{33}\right)^{-1} w_{4} M_{4}^{1} \mu+w_{4} M_{4}^{3} \mu+\text { h.o.t. }=0\right\}
$$

for $w_{4} M_{4}^{3} \mu>0$. So system (1.6) has a heteroclinic orbit consisting of $p_{3}$ and $p_{1}$ near $\Gamma_{1} \cup \Gamma_{3}$ for $\mu \in L_{4}^{2}(\mu)$. Next putting the expression of $s_{4}^{2^{*}}$ into the verification condition, it is equivalently $\left|\left(w_{2}^{31} M_{2}^{3} \mu+M_{2}^{1} \mu\right)\right| \ll\left|M_{4}^{1} \mu\right|^{1-\frac{1}{\alpha}}$.

b) As $\left|M_{4}^{1} \mu\right| \ll \max \left\{s_{4}^{\alpha},\left|\left(w_{2}^{31} M_{2}^{3} \mu+M_{2}^{1} \mu\right)\right| s_{4}\right\}$, Equation (3.2) is then

$$
w_{4}^{31}\left(w_{2}^{31} M_{2}^{3} \mu+M_{2}^{1} \mu\right) s_{4}-w_{4}^{33} \delta s_{4}^{\alpha}+\text { h.o.t. }=0,
$$

there is a small positive solution $s_{4}^{3^{*}}=\left(\frac{w_{4}^{31}\left(w_{2}^{31} M_{2}^{3} \mu+M_{2}^{1} \mu\right)}{w_{4}^{33} \delta}\right)^{\frac{1}{\alpha-1}}$. In the same way, we can get the surface $L_{4}^{3}(\mu)$ which is tangent to $L_{14}$ with the condition $\left|M_{4}^{1} \mu\right| \gg \mid\left(w_{2}^{31} M_{2}^{3} \mu+M_{2}^{1} \mu\right)^{1-\frac{1}{\alpha}}$, where

$$
L_{4}^{3}(\mu)=\left\{\mu: w_{4}^{13} \delta\left(w_{4}^{31}\left(w_{2}^{31} M_{2}^{3} \mu+M_{2}^{1} \mu\right)\right)^{\frac{\lambda_{2}^{2}}{\lambda_{2}^{2}-\lambda_{2}^{1}}}+\left(w_{4}^{33} \delta\right)^{\frac{\lambda_{2}^{2}}{\lambda_{2}^{2}-\lambda_{2}^{1}}} w_{4} M_{4}^{3} \mu+\text { h.o.t. }=0\right\}
$$

So system (1.6) has a heteroclinic orbit consisting of $p_{3}$ and $p_{1}$ in the region $\Gamma_{1} \cup \Gamma_{3}$ for $\mu \in L_{4}^{3}(\mu)$.

4) If $w_{4}^{33}>0, \mu \in B_{+}^{-}$or $w_{4}^{33}<0, \mu \in B_{-}^{+}$, without loss of generality, we discuss the case $w_{4}^{33}>0, \mu \in B_{+}^{-}$. There are $L(0, \mu)<N(0, \mu)$, $L_{s_{4}}^{\prime \prime}\left(s_{4}, \mu\right)-N_{s_{4}}^{\prime \prime}\left(s_{4}, \mu\right)=-w_{4}^{33} \alpha(\alpha-1) \delta s_{4}^{\alpha-2}+$ h.o.t. $<0$.

Set $L\left(\tilde{s}_{4}, \mu\right)-N\left(\tilde{s}_{4}, \mu\right)=H_{4}^{1}(\mu)$, where $\tilde{s}_{4}=\left(w_{4}^{31}\left(w_{4}^{33} \alpha \delta\right)^{-1}\left(w_{2}^{31} M_{2}^{3} \mu+M_{2}^{1} \mu\right)\right)^{\frac{1}{\alpha-1}}+$ h.o.t. is the solution of $L_{s_{4}}^{\prime}\left(s_{4}, \mu\right)-N_{s_{4}}^{\prime}\left(s_{4}, \mu\right)=0$ and

$$
\begin{aligned}
H_{4}^{1}(\mu)= & w_{4}^{31}\left(w_{2}^{31} M_{2}^{3} \mu+M_{2}^{1} \mu\right)\left(1-\frac{1}{\alpha}\right)\left(w_{4}^{31}\left(w_{4}^{33} \delta \alpha\right)^{-1}\left(w_{2}^{31} M_{2}^{3} \mu+M_{2}^{1} \mu\right)\right)^{\frac{1}{\alpha-1}} \\
& +w_{4} M_{4}^{1} \mu+\text { h.o.t.. }
\end{aligned}
$$

when $H_{4}^{1}(\mu)>0$, the straight line $L\left(s_{4}, \mu\right)$ intersects the curve $N\left(s_{4}, \mu\right)$ exactly at two points $0<s_{4}^{4^{*}}<\tilde{s}_{4}<s_{4}^{5^{*}}$, which means Equation (3.2) has two positive solutions. Therefore, system (1.6) has two heteroclinic orbits connecting $p_{3}$ and $p_{1}$ near $\Gamma_{1} \cup \Gamma_{3}$.

When $H_{4}^{1}(\mu)=0$, the equations $L_{s_{4}}^{\prime}\left(s_{4}, \mu\right)=N_{s_{4}}^{\prime}\left(s_{4}, \mu\right)$ and $L\left(s_{4}, \mu\right)=N\left(s_{4}, \mu\right)$ have the solution $\tilde{s}_{4}$, therefore the straight line $L\left(s_{4}, \mu\right)$ must be tangent to the curve $N\left(s_{4}, \mu\right)$ at the point $\tilde{s}_{4}$. Putting it into the second equation of (3.1) yields a surface $L_{4}^{4}(\mu)$ with a normal surface $\Sigma=\operatorname{span}\left\{M_{4}^{3}\right\}$ at $\mu=0$, where

$L_{4}^{4}(\mu)=\left\{\mu: w_{4}^{13} \delta\left(w_{4}^{31}\left(w_{2}^{31} M_{2}^{3} \mu+M_{2}^{1} \mu\right)\right)^{\frac{\lambda_{2}^{2}}{\lambda_{2}^{2}-\lambda_{2}^{11}}}+\left(w_{4}^{33} \alpha \delta\right)^{\frac{\lambda_{2}^{1}}{\lambda_{2}^{2}-\lambda_{2}^{1}}} w_{4} M_{4}^{3} \mu+\right.$ h.o.t. $\left.=0\right\}$ 
for $M_{4}^{3} \mu>0$. Then, system (1.6) has a 2-fold heteroclinic orbit connecting $p_{3}$ and $p_{1}$ near $\Gamma_{1} \cup \Gamma_{3}$.

When $H_{4}^{1}(\mu)<0$, the straight line $L_{2}\left(t_{2}, \mu\right)$ does not intersect the curve $N_{2}\left(t_{2}, \mu\right)$ in the half plane, then there is only the transversal heteroclnic orbit $\gamma_{3}(t)$ connecting $p_{2}$ and $p_{3}$ near $\Gamma_{2} \cup \Gamma_{3}$.

5) If $\mu \in\left\{\mu: M_{4}^{1} \mu+\right.$ h.o.t. $=M_{4}^{3} \mu+$ h.o.t. $\left.=0\right\}$, Equation (3.1) is

$$
\left\{\begin{array}{l}
w_{4}^{33} \delta s_{4}^{\frac{\lambda_{2}^{2}}{\lambda_{2}^{1}}}-w_{4}^{31} s_{4}\left(w_{2}^{31} M_{2}^{3} \mu+M_{2}^{1} \mu\right)=0 \\
w_{4}^{13} \delta s_{4}^{\frac{\lambda_{2}^{2}}{\lambda_{2}^{1}}}+w_{4}^{11} s_{4}\left(w_{2}^{31} M_{2}^{3} \mu+M_{2}^{1} \mu\right)=0 .
\end{array}\right.
$$

To solve the first equation of (3.5), there is

$$
s_{4}\left(w_{4}^{33} \delta s_{4}^{\alpha-1}-w_{4}^{31}\left(w_{2}^{31} M_{2}^{3} \mu+M_{2}^{1} \mu\right)\right)=0,
$$

we can get two solutions $s_{4}^{\prime}=0$ and $s_{4}^{\prime \prime}=\left(\frac{w_{4}^{31}\left(w_{2}^{31} M_{2}^{3} \mu+M_{2}^{1} \mu\right)}{w_{4}^{33} \delta}\right)^{\frac{1}{\alpha-1}}$ for $w_{4}^{31} w_{4}^{33}\left(w_{2}^{31} M_{2}^{3} \mu+M_{2}^{1} \mu\right)>0$. However, if $w_{4}^{31} w_{4}^{33}\left(w_{2}^{31} M_{2}^{3} \mu+M_{2}^{1} \mu\right)$, the above equation has only one zero solution. Equation (3.5) finally defines a surface $\overline{L_{4}^{3}}(\mu)$.

Putting the expression $s_{2}^{\prime \prime}$ into the second equation of (3.5) obtains the set of $\begin{aligned} \mu \text { as } & \left\{\mu \mid w_{4}^{13} \delta\left(w_{4}^{33} \delta\right)^{\frac{\lambda_{2}^{2}}{\lambda_{2}^{2}-\lambda_{2}^{1}}}\left(w_{4}^{31}\left(w_{2}^{31} M_{2}^{3} \mu+M_{2}^{1} \mu\right)\right)^{\frac{\lambda_{2}^{2}}{\lambda_{2}^{2}-\lambda_{2}^{1}}}\right. \\ & \left.+w_{4}^{11}\left(w_{4}^{33} \delta\right)^{\frac{\lambda_{2}^{1}}{\lambda_{2}^{2}-\lambda_{2}^{1}}}\left(w_{2}^{31} M_{2}^{3} \mu+M_{2}^{1} \mu\right)^{\frac{\lambda_{2}^{2}}{\lambda_{2}^{2}-\lambda_{2}^{1}}}=0,\left(w_{2}^{31} M_{2}^{3} \mu+M_{2}^{1} \mu\right) \neq 0\right\}, \text { that }\end{aligned}$ means the system of (6) coexists two types of heteroclinic orbit: a large-1 heteroclinc orbit connecting with $p_{3}$ and $p_{1}$, a heteroclinic orbit composed of two orbits which one orbit connects with $p_{3}$ and $p_{2}$ and the other orbit connects with $p_{2}$ and $p_{1}$ in the region $\Gamma_{1} \cup \Gamma_{3}$ as $\mu \in \bar{L}_{4}^{3}(\mu)$, where

$$
\bar{L}_{4}^{3}(\mu)=\left\{\mu: M_{4}^{1} \mu+\text { h.o.t. }=M_{4}^{3} \mu+\text { h.o.t. }=0, w_{4}^{31} w_{4}^{33}\left(w_{2}^{31} M_{2}^{3} \mu+M_{2}^{1} \mu\right)>0\right\} \text {. }
$$

Remark 3.1. The analysis of the third and fourth equations of (20) is similar to the above analysis process, so it will not be repeated here.

\subsection{Bifurcation Conclusions}

With the analysis above, we can get the following theorems about existence of the second and the third shape heterodimensional cycle and the large- 1 heteroclinic cycle under small perturbation.

Theorem 3.1. Under $\left(H_{1}\right)-\left(H_{4}\right)$ and Rank $\left(M_{1}^{1}, M_{1}^{3}, M_{4}^{1}, M_{4}^{3}\right) \geq 3$, as well as $\mu \in\left\{\mu: M_{4}^{1} \mu+\right.$ h.o.t. $=M_{4}^{3} \mu+$ h.o.t. $\left.=0\right\}$, there are the following conclusions:

1) If $\mu \in\left\{\mu: w_{4}^{33}>0, \mu \in B_{-}^{-}\right\}$or $\mu \in\left\{\mu: w_{4}^{33}<0, \mu \in B_{+}^{+}\right\}$, the system (1.6) exists the third shape heterodimensional cycle in the $(l-2)$-dimensional surface $L_{4}^{1}(\mu)$ with normal vector $M_{2}^{1}, M_{2}^{3}$ at $\mu=0$, where 


$$
L_{4}^{1}(\mu)=\left\{\mu: M_{4}^{1} \mu+\text { h.o.t. }=M_{4}^{3} \mu+\text { h.o.t. }=0, w_{4}^{31} w_{4}^{33}\left(w_{2}^{31} M_{2}^{3} \mu+M_{2}^{1} \mu\right) \leq 0\right\} .
$$

2) If $\mu \in\left\{\mu: w_{4}^{33}>0, \mu \in B_{+}^{-}\right\}\left(\mu \in\left\{\mu: w_{4}^{33}<0, \mu \in B_{-}^{+}\right\}\right)$, the system (1.6) exists the third shape heterodimensional cycle near $\Gamma$ as $\mu \in \tilde{L}_{4}^{4}$ and $0<|\mu| \ll 1$, where

$$
\tilde{L}_{4}^{4}=\left\{\mu: M_{4}^{1} \mu+\text { h.o.t. }=M_{4}^{3} \mu+\text { h.o.t. }=0, w_{4}^{31} w_{4}^{33}\left(w_{2}^{31} M_{2}^{3} \mu+M_{2}^{1} \mu\right) \leq 0, H_{4}^{1}<0\right\} .
$$

3) If $\mu \in\left\{\mu: w_{4}^{33}>0, \mu \in B_{-}^{+}\right\}$or $\mu \in\left\{\mu: w_{4}^{33}<0, \mu \in B_{+}^{-}\right\}$, there exists an $(l-1)$-dimensional surface

$$
\begin{gathered}
\hat{L}_{4}^{1}=\left\{\mu: M_{4}^{1} \mu+\text { h.o.t. }=M_{4}^{3} \mu+\text { h.o.t. }=0, w_{4}^{31} w_{4}^{33}\left(w_{2}^{31} M_{2}^{3} \mu+M_{2}^{1} \mu\right) \leq 0,\right. \\
\left.\mu \in L_{4}^{1}(\mu),\left|M_{4}^{1} \mu\right|=o\left(\left|w_{2}^{31} M_{2}^{3} \mu+M_{2}^{1} \mu\right|\right)\right\}
\end{gathered}
$$

with normal vector $M_{3}^{3}, M_{2}^{1}, M_{2}^{3}$ at $\mu=0$, which is tangent to the surface $L_{23}(\mu)$ at $\mu=0$, such that the system (1.6) has the second shape hetrodimensional cycle near $\Gamma$ as $\mu \in \hat{L}_{4}^{1}$ and $0<|\mu| \ll 1$.

4) If $\mu \in\left\{\mu: w_{4}^{33}>0, \mu \in B_{+}^{+}\right\}$or $\mu \in\left\{\mu: w_{4}^{33}<0, \mu \in B_{-}^{-}\right\}$, there exist two $(l-1)$-dimensional surfaces

$$
\begin{gathered}
\hat{L}_{4}^{2}=\left\{\mu: M_{4}^{1} \mu+\text { h.o.t. }=M_{4}^{3} \mu+\text { h.o.t. }=0, w_{4}^{31} w_{4}^{33}\left(w_{2}^{31} M_{2}^{3} \mu+M_{2}^{1} \mu\right) \leq 0,\right. \\
\left.\mu \in L_{4}^{2}(\mu),\left|\left(w_{2}^{31} M_{2}^{3} \mu+M_{2}^{1} \mu\right)\right| \ll\left|M_{4}^{1} \mu\right|^{1-\frac{1}{\alpha}}\right\}
\end{gathered}
$$

and

$$
\begin{gathered}
\hat{L}_{4}^{2}=\left\{\mu: M_{4}^{1} \mu+\text { h.o.t. }=M_{4}^{3} \mu+\text { h.o.t. }=0, w_{4}^{31} w_{4}^{33}\left(w_{2}^{31} M_{2}^{3} \mu+M_{2}^{1} \mu\right) \leq 0,\right. \\
\left.\mu \in L_{4}^{3}(\mu),\left|\left(w_{2}^{31} M_{2}^{3} \mu+M_{2}^{1} \mu\right)\right| \gg\left|M_{4}^{1} \mu\right|^{1-\frac{1}{\alpha}}\right\}
\end{gathered}
$$

such that the system (1.6) has the second shape heterodimensional cycle near $\Gamma$ as $\mu \in \hat{L}_{4}^{2}, \quad \mu \in \hat{L}_{4}^{2}$, respectively, and $0<|\mu| \ll 1$.

An alternative explanation for the existence of the second heterodimensional cycle is as follows. If there is an orbit starting from the section $S_{1}^{0}$ and arriving at the section $S_{3}^{1}$ that passes through the sections $S_{1}^{1}$ and $S_{3}^{0}$ with finite time without orienting to the saddle point $p_{2}$, we denote it by $\gamma_{1}(t, \mu)$. Similarly, we can define $\gamma_{4}(t, \mu)$ in this way. Set the time of the orbit $\gamma_{1}(t, \mu)$ from $S_{1}^{1}$ to $S_{3}^{0}$ to be $\tau_{2}$ and the time of $\gamma_{4}(t, \mu)$ from $S_{4}^{1}$ to $S_{2}^{0}$ to be $\tau_{4}$; and from $S_{k}^{0}$ to $S_{k}^{1}$ to be $\tilde{T}_{k}, k=1,2,3,4$, respectively. Moreover, system (1.6) still has solutions $\gamma_{j}(t, \mu), j=1,4$,

$$
\begin{aligned}
& \dot{\gamma}_{j}(t, \mu)=f\left(\gamma_{j}, \mu\right) \\
& \gamma_{j}\left(-T_{j}, \mu\right) \in S_{j}^{0}, \gamma_{j}\left(-T_{j}+\tilde{T}_{j}, \mu\right) \in S_{j}^{1}, \\
& \gamma_{1}\left(-T_{1}+\tilde{T}_{1}+\tau_{2}+\tilde{T}_{3}, \mu\right) \in S_{3}^{1}, \\
& \gamma_{4}\left(-T_{4}+\tilde{T}_{4}+\tau_{4}+\tilde{T}_{2}, \mu\right) \in S_{2}^{1},
\end{aligned}
$$




$$
\begin{aligned}
& \left\|\gamma_{1}\left(-T_{1}+\tilde{T}_{1}+\tau_{2}+\tilde{T}_{3}, \mu\right)-\gamma_{3}^{-}\left(T_{3}, \mu\right)\right\| \ll 1, \\
& \left\|\gamma_{4}\left(-T_{4}-\tilde{T}_{4}+\tau_{4}-\tilde{T}_{2}, \mu\right)-\gamma_{2}^{-}\left(T_{2}, \mu\right)\right\| \ll 1,
\end{aligned}
$$

Theorem 3.2. Suppose that $\left(H_{1}\right)-\left(H_{4}\right)$ hold and Rank $\operatorname{Rank}\left(M_{1}^{1}, M_{1}^{3}, M_{4}^{1}, M_{4}^{3}\right)=4$ there is an $(l-4)$-dimensional surface

$$
L_{14}=\left\{\mu: M_{1}^{1} \mu+\text { h.o.t. }=M_{1}^{3} \mu+\text { h.o.t. }=M_{4}^{1} \mu+\text { h.o.t. }=M_{4}^{3} \mu+\text { h.o.t. }\right\}
$$

with a normal plane $\Sigma_{23}=\operatorname{span}\left\{M_{1}^{1}, M_{1}^{3}, M_{4}^{1}, M_{4}^{3}\right\}$, such that system (1.6) has a unique double heteroclinic loop (" $\infty$ ") in the tubular neighborhood of $\Gamma$ as $\mu \in L_{24}, \quad 0<|\mu| \leq 1$.

Proof. As we explained above, $s_{2}=0$ in Equation (2.11) means the flying time of an orbit starting from $S_{1}^{1}$ to $S_{3}^{0}$ is infinite, that is, the orbit must go into the equilibrium $p_{2}$ and then leave, which corresponds to a heteroclinic orbit; and for $s_{4}=0$, it is similar. Hence, set $s_{2}=s_{4}=0$ in Equation (2.11), we have

$$
\left\{\begin{array}{l}
M_{1}^{1} \mu+\text { h.o.t. }=0, \\
M_{1}^{3} \mu+\text { h.o.t. }=0, \\
M_{4}^{1} \mu+\text { h.o.t. }=0, \\
M_{4}^{3} \mu+\text { h.o.t. }=0 .
\end{array}\right.
$$

If $\operatorname{Rank}\left(M_{1}^{1}, M_{1}^{3}, M_{4}^{1}, M_{4}^{3}\right)=4$, there is a codimension-4 surface with a normal plane spanned by $M_{1}^{1}, M_{1}^{3}, M_{4}^{1}, M_{4}^{3}$ as below

$$
L_{14}(\mu)=\left\{\mu: M_{1}^{1} \mu+\text { h.o.t. }=M_{1}^{3} \mu+\text { h.o.t. }=M_{4}^{1} \mu+\text { h.o.t. }=M_{4}^{3} \mu+\text { h.o.t. }=0\right\} .
$$

when $\mu \in L_{14}$, system (1.6) has four heteroclinic orbits connecting the equilibriums $p_{i}, i=1,2,3$, and they form an " $\infty$ "-type double heterodimensional cycle, or it says that the original heterodimensional cycle is preserved.

Corresponding, some new orbits $\gamma_{k}^{+}(t, \mu)$ (resp. $\left.\gamma_{k}^{-}(t, \mu)\right) \quad(k=1,2,3,4)$ appear from unstable (resp. stable) manifold of the equilibrium $p_{i}(i=1,2,3)$ of system (1.6) with the following properties,

$$
\begin{aligned}
& \dot{\gamma}_{k}^{ \pm}=f\left(\gamma_{k}^{ \pm}, \mu\right) ; \\
& \gamma_{1}^{+}(t, \mu) \in W_{1}^{u}\left(p_{1}\right), \quad \gamma_{1}^{-}(t, \mu) \in W_{2}^{s}\left(p_{2}\right), \\
& \gamma_{2}^{+}(t, \mu) \in W_{2}^{u}\left(p_{2}\right), \quad \gamma_{2}^{-}(t, \mu) \in W_{1}^{s}\left(p_{1}\right), \\
& \gamma_{3}^{+}(t, \mu) \in W_{2}^{u}\left(p_{2}\right), \quad \gamma_{3}^{-}(t, \mu) \in W_{3}^{s}\left(p_{3}\right), \\
& \gamma_{4}^{+}(t, \mu) \in W_{3}^{u}\left(p_{3}\right), \quad \gamma_{4}^{-}(t, \mu) \in W_{2}^{s}\left(p_{2}\right), \\
& \gamma_{k}^{ \pm}(t, 0)=\gamma_{k}(t), \\
& \gamma_{k}^{+}\left(-T_{k}, \mu\right) \in S_{k}^{0}, \\
& \gamma_{k}^{+}\left(-T_{k}+\tilde{T}_{k}, \mu\right), \gamma_{k}^{-}\left(T_{k}, \mu\right) \in S_{k}^{1}, \\
& \left\|\gamma_{3}^{+}\left(-T_{3}+\tilde{T}_{3}, \mu\right)-\gamma_{3}^{-}\left(T_{k}, \mu\right)\right\|=0(k=1,4) \\
& \left\|\gamma_{k}^{+}\left(-T_{k}+\tilde{T}_{k}, \mu\right)-\gamma_{k}^{-}\left(T_{k}, \mu\right)\right\| \ll 1(k=2,3),
\end{aligned}
$$

where $W_{i}^{s}\left(p_{i}\right)$ and $W_{i}^{u}\left(p_{i}\right)$ are the stable and unstable manifolds of the 
equilibrium $p_{i}$, because the original heteroclinic trajectory $\gamma_{3}(t)$ is obtained as a transversal intersection of 2-dimensional manifolds, which is a structurally stable situation. After a small perturbation, such an intersection is preserved. That is, the gap $\left\|\gamma_{3}^{+}\left(-T_{3}+\tilde{T}_{3}, \mu\right)-\gamma_{3}^{-}\left(T_{3}, \mu\right)\right\|=0$. As well as, if the gap $\left\|\gamma_{k}^{+}\left(-T_{k}+\tilde{T}_{k}, \mu\right)-\gamma_{k}^{-}\left(T_{k}, \mu\right)\right\|=0 \quad(k=1,2,4)$ in $S_{k}^{1}$, it means that the original double heterodimensional cycles are kept (see Figure 3).

Where $\gamma_{k}^{ \pm}(t, \mu) \quad(k=2,4)$, and $\gamma_{3}^{-}(t, \mu)$ still meet Equation (3.1). Clearly system (1.6) has the second shape heterodimensional cycle, if the gaps $\left\|\gamma_{1}\left(-T_{1}+\tilde{T}_{1}+\tau_{2}+\tilde{T}_{3}, \mu\right)-\gamma_{3}^{-}\left(T_{3}, \mu\right)\right\|=0, \quad\left\|\gamma_{2}^{-}\left(-T_{2}, \mu\right)-\gamma_{2}^{+}\left(T_{2}+\tilde{T}_{2}, \mu\right)\right\|=0 \quad$ (see Figure 4).

Remark 3.2. The second heterodimensional cycle consists of two saddles of (1.2) type and one saddle of (2.1) type and is composed of one big orbit linking $p_{1}, p_{3}$ and two orbits linking $p_{3}, p_{2}$ and $p_{2}, p_{1}$ respectively (see Figure 3 ).

Remark 3.3. As for the other theorem of the similar second shape heterdimensional cycle which consists of two saddles of (1.2) type and one saddle of (2.1) type and is composed of one big orbit linking $p_{1}, p_{3}$ and two orbits linking $p_{3}, p_{2}$ and $p_{2}, p_{1}$ respectively is analogous to theorem 3.1 , so it will not be repeated here.

Theorem 3.3. Suppose $\left(H_{1}\right)-\left(H_{4}\right)$ are valid and $\operatorname{Rank}\left(M_{1}^{1}, M_{1}^{3}, M_{4}^{1}, M_{4}^{3}\right) \geq 3$, there are the following conclusions.

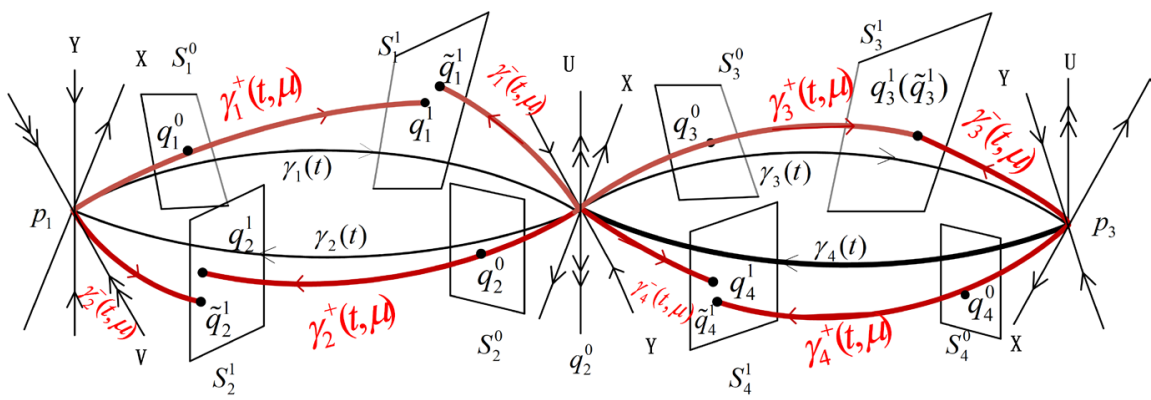

Figure 3. The gap $\left\|\gamma_{k}^{+}\left(-T_{k}+\tilde{T}_{k}, \mu\right)-\gamma_{k}^{-}\left(T_{k}, \mu\right)\right\|=0(k=1,2,4)$ in the figure, the original double heterodimensional cycles exists.

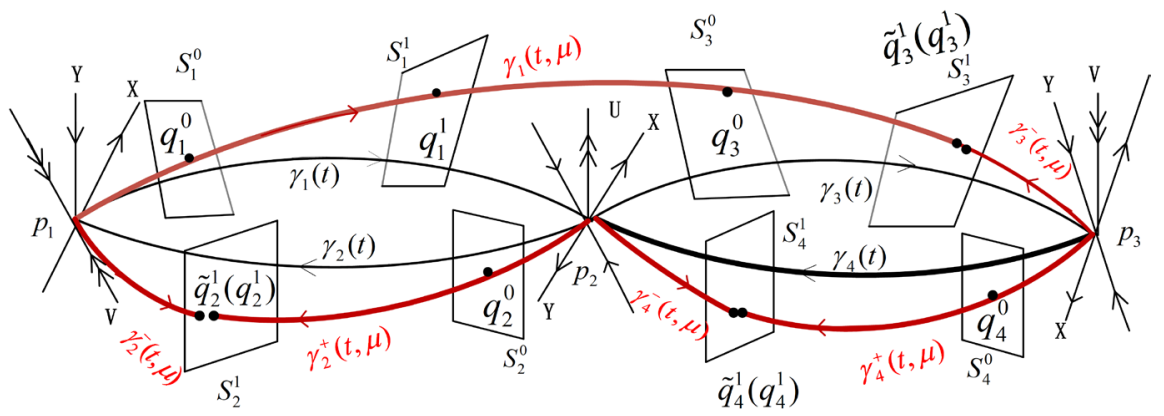

Figure 4. The gap $\left\|\gamma_{1}\left(-T_{1}+\tilde{T}_{1}+\tau_{2}+\tilde{T}_{3}, \mu\right)-\gamma_{3}^{-}\left(-T_{3}, \mu\right)\right\|=0$,

$\left\|\gamma_{2}^{-}\left(-T_{2}, \mu\right)-\gamma_{2}^{+}\left(T_{2}+\tilde{T}_{2}, \mu\right)\right\|=0$ in the figure, there is the second heterodimensional cycle. 
1) If $\mu \in\left\{\mu: w_{1}^{33}>0, w_{4}^{33}>0, \mu \in B_{-}^{+}, \mu \in N_{-}^{+}\right\}$or $\mu \in\left\{\mu: w_{1}^{33}<0, w_{4}^{33}<0, \mu \in B_{+}^{-}, \mu \in N_{+}^{-}\right\}$, there exists an (l-2)-dimensional surface

$$
\begin{aligned}
H_{24}^{1}(\mu)= & \left\{\mu:\left|M_{4}^{1} \mu\right|=o\left(\left|w_{2}^{31} M_{2}^{3} \mu+M_{2}^{1} \mu\right|\right),\left|M_{1}^{1} \mu\right|=o\left(\left|w_{3}^{32} M_{3}^{3} \mu+M_{3}^{1} \mu\right|\right),\right. \\
& \left.\mu \in L_{2}^{1}(\mu) \cap L_{4}^{1}(\mu), L_{2}^{1}(\mu) \cap L_{4}^{1}(\mu) \neq \varnothing\right\}
\end{aligned}
$$

with normal vector $M_{1}^{1}, M_{4}^{1}$ at $\mu=0$, which is tangent to the surface $L_{14}(\mu)$, then the system (1.6) has a 1-fold large-1 heteroclinic cycle near $\Gamma$ as $\mu \in H_{24}^{1}(\mu)$ and $0<|\mu| \ll 1$, where

$$
L_{2}^{1}(\mu)=\left\{\mu: w_{1}^{13} \delta\left(\frac{w_{1} M_{1}^{1} \mu}{w_{1}^{31} w_{3}^{32} M_{3}^{3} \mu+w_{1}^{31} M_{3}^{1} \mu}\right)^{\frac{\lambda_{2}^{2}}{\lambda_{2}^{1}}}-w_{1}^{11}\left(w_{1}^{31}\right)^{-1} w_{1} M_{1}^{1} \mu+w_{1} M_{1}^{3} \mu+\text { h.o.t. }=0\right\} .
$$

2) If $\mu \in\left\{\mu: w_{1}^{33}>0, w_{4}^{33}>0, \mu \in B_{-}^{+}, \mu \in N_{-}^{+}\right\}$or

$\mu \in\left\{\mu: w_{1}^{33}<0, w_{4}^{33}<0, \mu \in B_{+}^{-}, \mu \in N_{+}^{-}\right\}$, there exists two (l-2)-dimensional surface

$$
\begin{aligned}
H_{24}^{2}(\mu)= & \left\{\mu:\left|M_{4}^{1} \mu\right| \ll\left|\left(w_{2}^{31} M_{2}^{3} \mu+M_{2}^{1} \mu\right)\right|^{1-\frac{1}{\alpha}},\left|M_{1}^{1} \mu\right| \ll\left|\left(w_{3}^{32} M_{3}^{3} \mu+M_{3}^{1} \mu\right)\right|^{1-\frac{1}{\alpha}},\right. \\
& \left.\mu \in L_{2}^{2}(\mu) \cap L_{4}^{2}(\mu), L_{2}^{2}(\mu) \cap L_{4}^{2}(\mu) \neq \varnothing\right\}
\end{aligned}
$$

and

$$
\begin{aligned}
H_{24}^{3}(\mu)= & \left\{\mu:\left|\left(w_{2}^{31} M_{2}^{3} \mu+M_{2}^{1} \mu\right)\right|^{1-\frac{1}{\alpha}} \ll\left|M_{4}^{1} \mu\right|,\left|\left(w_{3}^{32} M_{3}^{3} \mu+M_{3}^{1} \mu\right)\right|^{1-\frac{1}{\alpha}} \ll\left|M_{1}^{1} \mu\right|,\right. \\
& \left.\mu \in L_{2}^{3}(\mu) \cap L_{4}^{3}(\mu), L_{2}^{3}(\mu) \cap L_{4}^{3}(\mu) \neq \varnothing\right\}
\end{aligned}
$$

both with normal vector $M_{1}^{3}, M_{4}^{3}$ at $\mu=0$, which both are tangent to the surface $L_{23}(\mu)$, then the system (1.6) has a 1-fold large-1 heteroclinic cycle near $\Gamma$ as $\mu \in H_{24}^{2}(\mu), \quad \mu \in H_{24}^{3}(\mu)$, respectively, and $0<|\mu| \ll 1$, where

$$
L_{2}^{2}(\mu)=\left\{\mu: w_{1}^{11}\left(-\frac{w_{1} M_{1}^{1} \mu}{w_{1}^{33} \delta}\right)^{\frac{\lambda_{2}^{1}}{\lambda_{2}^{2}}}\left(w_{3}^{32} M_{3}^{3} \mu+M_{3}^{1} \mu\right)+w_{1}^{13}\left(w_{1}^{33}\right)^{-1} w_{1} M_{1}^{1} \mu-w_{1} M_{1}^{3} \mu+\text { h.o.t. }=0\right\}
$$

and

$$
L_{2}^{3}(\mu)=\left\{\mu: w_{1}^{13} \delta\left(w_{1}^{11}\left(w_{3}^{32} M_{3}^{3} \mu+M_{3}^{1} \mu\right)\right)^{\frac{\lambda_{2}^{2}}{\lambda_{2}^{2}-\lambda_{2}^{1}}}-\left(w_{1}^{33} \delta\right)^{\frac{\lambda_{2}^{2}}{\lambda_{2}^{2}-\lambda_{2}^{1}}} w_{1} M_{1}^{3} \mu+\text { h.o.t. }=0\right\} .
$$

3) If $\mu \in\left\{\mu: w_{1}^{33}>0, \mu \in B_{+}^{-}, w_{4}^{33}>0, \mu \in N_{+}^{-}\right\}$, and $H_{2}^{1}>0, H_{4}^{1}>0$, the system (1.6) has two 1-fold large-1 heteroclinic cycles near $\Gamma$, where

$$
\begin{aligned}
H_{2}^{1}(\mu)= & w_{1}^{11}\left(w_{3}^{32} M_{3}^{3} \mu+M_{3}^{1} \mu\right)\left(1-\frac{1}{\alpha}\right)\left(w_{1}^{11}\left(w_{1}^{33} \delta \alpha\right)^{-1}\left(w_{3}^{32} M_{3}^{3} \mu+M_{3}^{1} \mu\right)\right)^{\frac{1}{\alpha-1}} \\
& +w_{1} M_{1}^{1} \mu+\text { h.o.t. }
\end{aligned}
$$




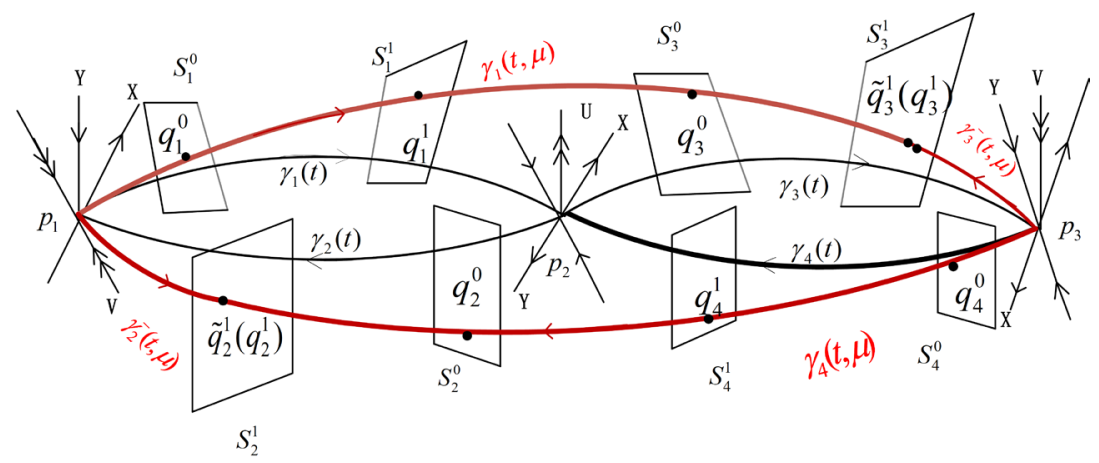

Figure 5. The gap $\left\|\gamma_{1}\left(-T_{1}+\tilde{T}_{1}+\tau_{2}+\tilde{T}_{3}, \mu\right)-\gamma_{3}^{-}\left(T_{3}, \mu\right)\right\|=0$,

$\left\|\gamma_{4}\left(-T_{4}+\tilde{T}_{4}+\tau_{4}+\tilde{T}_{2}, \mu\right)-\gamma_{2}^{-}\left(T_{2}, \mu\right)\right\|=0$ in the figure, there is the large 1-heteroclinic cycle.

4) If $w_{1}^{33}>0, w_{4}^{33}>0, \mu \in B_{-}^{+}, \mu \in N_{-}^{+}$, there exists a (l-2)-dimensional surface $H_{24}^{6}$ with normal vector $M_{1}^{3}, M_{4}^{3}$, which is tangent to the surface $L_{14}(\mu)$ at $\mu=0$, where

$$
H_{24}^{4}(\mu)=\left\{\mu: H_{2}^{1}=0, H_{4}^{1}=0, \mu \in L_{2}^{4} \cap L_{4}^{4}, L_{2}^{4} \cap L_{4}^{4} \neq \varnothing\right\},
$$

then the system (1.6) has one 2-fold large-1 heteroclinic cycles near $\Gamma$ for $\mu \in H_{24}^{4}$, where

$L_{2}^{4}(\mu)=\left\{\mu: w_{1}^{13} \delta\left(w_{1}^{11}\left(w_{3}^{32} M_{3}^{3} \mu+M_{3}^{1} \mu\right)\right)^{\frac{\lambda_{2}^{2}}{\lambda_{2}^{2}-\lambda_{2}^{1}}}+\left(w_{1}^{33} \alpha \delta\right)^{\frac{\lambda_{2}^{1}}{\lambda_{2}^{2}-\lambda_{2}^{1}}} w_{1} M_{1}^{3} \mu+\right.$ h.o.t. $\left.=0\right\}$.

For the alternative explanation from the gaps for the existence of the large-1 heteroclinic cycle is the following. If $\gamma_{k}(t, \mu)(k=1,4)$, and $\gamma_{k}^{-}(t, \mu)(k=2,3)$ still meet Equation (3.2) and (3.1). Clearly system (1.6) has a large 1-heteroclinic cycle composed of two big orbits linking $p_{1}, p_{3}$ and $p_{3}, p_{1}$ of (1.2) type respectively, if the gaps $\left\|\gamma_{1}\left(-T_{1}+\tilde{T}_{1}+\tau_{2}+\tilde{T}_{3}, \mu\right)-\gamma_{3}^{-}\left(T_{3}, \mu\right)\right\|=0$, $\left\|\gamma_{4}\left(-T_{4}-\tilde{T}_{4}+\tau_{4}-\tilde{T}_{2}, \mu\right)-\gamma_{2}^{-}\left(T_{2}, \mu\right)\right\|=0$ (see Figure 5).

\section{Acknowledgements}

We gratefully acknowledge the reviewers for their patience in reading the first draft of this paper.

\section{Funding}

The authors were supported by National Natural Science Foundation of China (Grant No. 11871022).

\section{Conflicts of Interest}

The authors declare that they have no competing interests.

\section{References}

[1] Zhu, D.M. (1998) Problems in Homoclinic Bifurcation with Higher Dimensions. 
Acta Mathematica Sinica, 14, 341-352. https://doi.org/10.1007/BF02580437

[2] Han, M.A. and Zhu, H.P. (2007) The Loop Quantities and Bifurcations of Homoclinic Loops. Journal of Differential Equations, 234, 339-359.

https://doi.org/10.1016/j.jde.2006.11.009

[3] Homburg, A.J. and Sandstede, B. (2010) Chapter 8: Homoclinic and Heteroclinic Bifurcations in Vector Fields. In: Broer, H., Takens, F. and Hasselblatt, B., Eds., Handbook of Dynamical Systems, Vol. 3, Elsevier B.V., Amsterdam, 379-524. https://doi.org/10.1016/S1874-575X(10)00316-4

[4] Geng, F.J., Lin, X.B. and Liu, X.B. (2019) Chaotic Traveling Wave Solutions in Coupled Chua's Circuits. Journal of Dynamics and Differential Equations, 31, 1373-1396. https://doi.org/10.1007/s10884-017-9631-1

[5] Zilburg, A. and Rosenau, P. (2018) Multi-Dimensional Compactons and Compact Vortices. Journal of Physics A: Mathematical and Theoretical, 51, Article ID: 395201. https://doi.org/10.1088/1751-8121/aad874

[6] Zhang, H.Y. and Zhang, K.L. (2019) Bifurcations of Traveling Wave Solutions for a Class of dKdV-Equation. Acta Mathematica Sinica, English Series, 39, 327-330.

[7] Newhouse, S. and Palis, J. (1973) Bifurcations of Morse-Smale Dynamical Systems. Dynamical Systems, 8, 303-366. https://doi.org/10.1016/B978-0-12-550350-1.50030-8

[8] Rademacher, J.D.M. (2005) Homoclinic Orbits near Heteroclinic Cycles with One Equilibrium and One Periodic Orbit. Journal of Differential Equations, 218, 390-443. https://doi.org/10.1016/j.jde.2005.03.016

[9] Bykov, V.V. (1993) The Bifurcations of Separatrix Contours and Chaos. Physica D, 62, 290-299. https://doi.org/10.1016/0167-2789(93)90288-C

[10] Liu, D., Ruan, S.G. and Zhu, D.M. (2011) Nongeneric Bifurcations near Heterodimensional Cycles with Inclination flip in $\mathrm{R}^{4}$. Discrete \& Continuous Dynamical Systems-S, 4, 1511-1532. https://doi.org/10.3934/dcdss.2011.4.1511

[11] Xu, Y.C. and Zhu, D.M. (2010) Bifurcations of Heterodimensional Cycles with One Orbit Flip and One Inclination Flip. Nonlinear Dynamics, 60, 1-13.

https://doi.org/10.1007/s11071-009-9575-Z

[12] Lu, Q.Y., Qiao, Z.Q., Zhang, T.S. and Zhu, D.M. (2010) Heterodimensional Cycle Bifurcation with Orbit-Flip. International Journal of Bifurcation and Chaos, 20, 491-508. https://doi.org/10.1142/S0218127410025569

[13] Lu, Q.Y., Zhu, D.M. and Geng, F.J. (2011) Weak Type Heterodimensional Cycle Bifurcation with Orbit-Flip. Science China Mathematics, 54, 1175-1196. https://doi.org/10.1007/s11425-011-4167-Z

[14] Yan, T.J. (2012) Bifurcation of Heterodimensional Cycle with Orbit Flip and Inclination Flip. Master Thesis, East Chins Normal University, Shanghai.

[15] Liu, X.B., Wang, X.F. and Wang, T. (2018) Nongeneric Bifurcations near a Nontransversal Heterodimensional Cycle. Chinese Annals of Mathematics, Series B, 39, 111-128. https://doi.org/10.1007/s11401-018-1055-7

[16] Zhang, T.S. and Zhu, D.M. (2006) Homoclinic Bifurcation of Orbit Flip with Resonant Principal Eigenvalues. Acta Mathematica Sinica, 22, 855-864.

https://doi.org/10.1007/s10114-005-0581-x

[17] Zhang, T.S. and Zhu, D.M. (2007) Bifurcations of Homoclinic Orbit Connecting Two Nonleading Eigendirections. International Journal of Bifurcation and Chaos, 17, 823-836. https://doi.org/10.1142/S0218127407017574

[18] Jin, Y.L. and Zhu, D.M. (2002) Bifurcations of Rough Heteroclinic Loops with 
Three Saddle Points. Acta Mathematica Sinica, 18, 199-208. https://doi.org/10.1007/s101140100139

[19] Zhang, W.P. (2007) Bifurcation of Double Homoclinic Loops in Four Dimensional Systems and Problems of Periodic Solutions in Population Dynamics. Ph.D. Thesis, East China Normal University, Shanghai.

[20] Geng, F.J. and Zhao, J.F. (2014) Bifurcations of Orbit and Inclination Flips Heteroclinic Loop with Nonhyperbolic Equilibria. The Scientific World Journal, 2014, Article ID: 585609. https://doi.org/10.1155/2014/585609

[21] Jin, Y., Zhu, X., Guo, Z., Xu, H., Zhang, L. and Ding, B. (2014) Bifurcations of Nontwisted Heteroclinic Loop with Resonant Eigenvalues. The Scientific World Journal, 2014, Article ID: 716082. https://doi.org/10.1155/2014/716082

[22] Jin, Y., Yang, S., Liu, Y., Xie, D. and Zhang, N. (2017) Bifurcations of Heteroclinic Loop with Twisted Conditions. International Journal of Bifurcation and Chaos, 27, Article ID: 1750120. https://doi.org/10.1142/S0218127417501206

[23] Jin, Y., Zhu, X., Liu, Y., Xu, H. and Zhang, N. (2018) Bifurcations of Twisted Heteroclinic Loop with Resonant Eigenvalues. Nonlinear Dynamics, 92, 557-573.

https://doi.org/10.1007/s11071-018-4075-7

[24] Jin, Y., Xu, H., Gao, Y.R., Zhao, X. and Xie, D. (2016) Bifurcations of Resonant Double Homoclinic Loops for Higher Dimensional Systems. Journal of Mathematical and Computational Science, 16, 165-177. https://doi.org/10.22436/jmcs.016.02.05

[25] Manna, K. and Banerjee, M. (2019) Stability of Hopf-Bifurcating Limit Cycles in a Diffusion-Driven Prey-Predator System with Allee Effect and Time Delay. Mathematical Biosciences and Engineering, 16, 2411-2446.

https://doi.org/10.3934/mbe.2019121 